\title{
THE
}

$12-7-2012$

\section{Ultracold Neutron Depolarization in Magnetic Bottles}

\author{
A. Steyerl \\ University of Rhode Island, a_steyerl@uri.edu \\ Charles Kaufman \\ University of Rhode Island, ckaufman@uri.edu \\ Gerhard Müller \\ University of Rhode Island, gmuller@uri.edu \\ S. S. Malik \\ University of Rhode Island, smalik@uri.edu
}

A. M. Desai

University of Rhode Island

Follow this and additional works at: https://digitalcommons.uri.edu/phys_facpubs

Terms of Use

All rights reserved under copyright.

\section{Citation/Publisher Attribution}

Steyerl, A., Kaufman, C., Muller, G., Malik, S. S., \& Desai, A. M. (2012). Ultracold Neutron Depolarization in Magnetic Bottles. Physical Review C, 86(6), 065501. doi: 10.1103/PhysRevC.86.065501

Available at http://dx.doi.org/10.1103/PhysRevC.86.065501

This Article is brought to you for free and open access by the Physics at DigitalCommons@URI. It has been accepted for inclusion in Physics Faculty Publications by an authorized administrator of DigitalCommons@URI. For more information, please contact digitalcommons-group@uri.edu. 


\title{
Ultracold neutron depolarization in magnetic bottles
}

\author{
A. Steyerl, ${ }^{*}$ C. Kaufman, G. Müller, S. S. Malik, and A. M. Desai \\ Department of Physics, University of Rhode Island, Kingston, Rhode Island 02881, USA
}

(Received 28 September 2012; published 7 December 2012)

\begin{abstract}
We analyze the depolarization of ultracold neutrons confined in a magnetic field configuration similar to those used in existing or proposed magnetogravitational storage experiments aiming at a precise measurement of the neutron lifetime. We use an extension of the semiclassical Majorana approach as well as an approximate quantum mechanical analysis, both pioneered by Walstrom et al. [Nucl. Instrum. Methods Phys. Res. A 599, 82 (2009)]. In contrast with this previous work we do not restrict the analysis to purely vertical modes of neutron motion. The lateral motion is shown to cause the predominant depolarization loss in a magnetic storage trap. The system studied also allowed us to estimate the depolarization loss suffered by ultracold neutrons totally reflected on a nonmagnetic mirror immersed in a magnetic field. This problem is of preeminent importance in polarized neutron decay studies such as the measurement of the asymmetry parameter $A$ using ultracold neutrons, and it may limit the efficiency of ultracold neutron polarizers based on passage through a high magnetic field.
\end{abstract}

DOI: 10.1103/PhysRevC.86.065501

PACS number(s): 28.20.-v, 14.20.Dh, 21.10.Tg

\section{INTRODUCTION}

The neutron lifetime $\tau_{\mathrm{n}}$ is an important parameter in tests of the standard model of particle physics. It also affects the rate of helium production in the early universe and the energy production in the sun. The current Particle Data Group (PDG) average is $\tau_{\mathrm{n}}=880.1 \pm 1.1 \mathrm{~s}$ [1]. However, the value of one experiment [2], which reported the lowest measurement uncertainty of $\sim 0.8 \mathrm{~s}$, is $\sim 3.5 \mathrm{~s}$ lower than the bulk of other data in the PDG collection [3-8], that are grouped consistently around $882.0 \mathrm{~s}( \pm 1.0 \mathrm{~s})$ [9]. Therefore the actual uncertainty of $\tau_{\mathrm{n}}$ to be used in cosmological calculations may be of the order of $2 \mathrm{~s}$ or more. As a possible way of advancing this field, storage of polarized ultracold neutrons (UCNs) in a magnetic trap has been pioneered by Paul et al. [10] and is currently being pursued vigorously by several groups worldwide [11-15]. One advantage of magnetic UCN storage versus storage in material bottles, the method used in a number of previous neutron lifetime measurements $[3,4,6,8]$, is the potential absence of losses due to effects other than $\beta$ decay. There are no wall losses, the slow loss due to quasistable orbits is serious but believed to be manageable by avoiding regular orbits [14], and the potential loss due to depolarization, defined as spin flip relative to the local field direction, is commonly assumed to be negligible. For systems using permanent magnets the question of gradual demagnetization over time appears to have found little attention so far.

Until recently UCN depolarization estimates [16,17] were based on Majorana's quasiclassical result of 1932 [18] for a free polarized particle with magnetic moment moving with constant velocity vector through a nonuniform static magnetic field of specific form. Only its spin state was assumed to be affected by the magnetic field. This model predicted a depolarization probability $D=\exp \left(-\pi \omega_{L} / 2 \omega\right)$ for one passage through the field. This value decreases exponentially with the adiabaticity parameter $\omega_{L} / \omega$, where $\omega$ is the frequency of rotation of the field as seen from the reference frame of

*asteyerl@mail.uri.edu the moving particle, in the critical region where the field rotates fastest while the magnitude $B$ of the magnetic field may be small. $\omega_{L}$ is the Larmor frequency. For magnetic field parameters as currently used or proposed for UCN storage, $D$ would be of order $\exp \left(-10^{6}\right)$, thus immeasurably small. Recently, Walstrom et al. [14] pointed out that the values of $D$ for confined, rather than freely moving, neutrons are much larger. For a UCN moving along a vertical path in the storage system proposed by them, $D$ was estimated to be in the range $D \sim 10^{-20}$ to $10^{-23}$. This is much larger than the Majorana value but still negligible in any actual or projected neutron lifetime experiment.

Using a simplified model of magnetic field distribution we extend the theory of [14] to include arbitrary UCN motion with both vertical and horizontal velocity components, confined to the vertical space between upper and lower turning points that depend only on the UCN energy for vertical motion. In our model (introduced in Sec. II) the magnetic field magnitude $B$ is uniform within any horizontal plane, so there is no horizontal component of magnetic force. Therefore the neutron moves with constant velocity in the horizontal $z$ and $x$ directions. We show that $D$ could reach a level approaching the tolerance limit for a high precision neutron lifetime measurement unless precautions are taken. As is well known the most critical issue is the choice of a stabilization field perpendicular to the magnetic mirror field, of sufficient strength so that the depolarization rate will be negligible in a neutron lifetime experiment.

Our model field is close to the "bathtub configuration" of Ref. [14] but the lateral confinement of UCNs, achieved there by double curvature of the magnetic mirror, is simulated differently. The magnetic mirror is horizontal and extends to infinity in both lateral dimensions. However, one could imagine the presence of ideal vertical mirrors reflecting the UCNs back and forth in the horizontal directions without any change in the analysis.

More specifically, we use an infinite ideal planar Halbach array [19], which is free of the field ripples present in actual realizations [14]. In the design of Ref. [14], the ripples are 
important only within about $1 \mathrm{~mm}$ of the surface of the magnets. This region is not reached by the UCNs whose maximum energy (for vertical motion) is $\sim 45 \mathrm{neV}$ (for the parameters in Ref. [14]), since they reverse their flight direction before entering this zone.

Using this model of field distribution we have also studied the problem of depolarization of UCNs in reflection from a nonmagnetic mirror immersed in a nonuniform magnetic field. This question is important as a mechanism that may limit the efficiency of UCN polarizers based on transmission through a magnetic field. For a sufficiently strong field, neutrons in only one spin state can pass the field to proceed to the experiment. Otherwise they are reflected. However, following the polarizer the UCNs are usually reflected on trap or guide walls exposed to the stray field of the polarizer and thus may lose their $100 \%$ polarization if the reflection process involves depolarization. Moreover, a possible depolarization on nonmagnetic trap walls in a magnetic field is highly relevant in measurements of the neutron decay asymmetry parameter $A$ using ultracold neutrons $[20,21]$. This problem has first been investigated by Pokotilovski [17] who used an adaptation of the Majorana model to the reflection geometry. In the present work we study certain aspects of this problem by imagining a horizontal lossless nonmagnetic UCN mirror inserted at a variable height into the magnetogravitational storage space. The net depolarization per bounce on this mirror will be compared to the depolarization for one bounce in the magnetic field in the absence of the mirror to obtain an estimate for the depolarization effect of the mirror.

The topic of UCN depolarization in magnetic storage or in mirror reflection in a magnetic field raises interesting questions of quantum interpretation. Figure 3 of Ref. [14] and our Fig. 2 (to be discussed in Sec. III B2) show the probability for the neutron to be in the spin-flipped state (relative to the field direction) as a function of position of the neutron as it moves through the magnetic storage space. The curve is strongly peaked at the critical level where the field rotates fastest in the reference frame of the moving neutron. This behavior is the same as displayed by the Majorana result [18] (where it is more difficult to deduce since the author used a quantization axis fixed in space rather than rotating together with the field). In a semiclassical interpretation, as the UCN starts moving from one turning point, say the upper one, down toward the lower one, the spin vector rotates away from the quantization axis (which was chosen parallel to the local magnetic field vector in Ref. [14]). It reaches a certain maximum angle around the critical zone; then this rotation is reversed and ends at a much smaller value at the next turning point for UCN motion. This indicates that an analyzer of neutron polarization placed at different heights would show a variation of depolarization by many orders of magnitude ( $>8$ decades for the example shown in Fig. 3 of Ref. [14]) over the vertical range of the storage space. The depolarization rate expected for an actual UCN magnetic storage experiment, without any polarization analyzer intersecting the beam, is determined by the current of UCNs in the "wrong" spin state, i.e., of high-field seekers leaving the system at the lower and upper turning points while the "correct" (high-field repelled) state is reflected and returns to the storage space. This association of net depolarization with loss currents is consistent with the following interpretation: At the turning points a measurement is performed (in the sense of quantum mechanics), conceptually by neutron detectors placed just below the bottom and just above the top of the storage region for a given UCN energy for vertical motion. These detectors would intersect the UCNs in the "wrong" spin state as they exit the storage system. In the Copenhagen interpretation, such a measurement (actual or hypothetical) resets the UCN wave function to a pure state of high-field repelled neutrons. The spin state then evolves as described by the spin-dependent Schrödinger equation (or its semiclassical analog) until the next "measurement" takes place at the following turning point and the process of wave collapse and wave evolution is repeated. Alternative interpretations are conceivable but we will use the picture outlined above.

Following Ref. [14] we use the Wentzel-KramersBrillouin (WKB) approximation to solve the spin-dependent Schrödinger equation. This appears justified since the spatial variation of field variables (gravitational potential and magnetic field $\mathbf{B}$ ) is much slower than the variation of UCN wave function. The scales are of order $\mathrm{cm}$ for gravity and $\mathbf{B}$, and of order $\mu \mathrm{m}$ or less for the neutron wavelength.

We are aware of the fact that an exact treatment of UCN depolarization in magnetic storage may involve quantum electrodynamics since the moving neutron, in its reference frame, is affected by a time-dependent electromagnetic field, i.e., by low-energy photons. We will also neglect temporal fluctuations of the field due to mechanical vibrations or, if electromagnets are used for field generation, AC components of the current supply. We are not aware of any work on time-dependent effects of this kind in magnetic UCN storage.

\section{MAGNETIC FIELD DISTRIBUTION}

We consider a Halbach array [19] of permanent magnets of thickness $d$ covering the infinite $(z x)$ plane, where the $x$ axis points to the right, the $y$ axis points up (against gravity) and the $z$ axis toward the front (Fig. 1). We choose $y=0$ at the upper magnet surface and will closely follow the description in Ref. [14], apart from this choice of system of coordinates. This choice will allow us to use the Pauli matrices in their standard form.

In the limit of infinitely fine division of magnet blocks in the $x$ direction, let the magnetization vector have a constant magnitude $M_{0}$ but, viewed along the positive $z$ direction, rotate clockwise in the $(x y)$ plane with periodicity $L=2 \pi / K$ in $x$ direction:

$$
\mathbf{M}(x)=M_{0}(\hat{\boldsymbol{x}} \cos K x+\hat{\boldsymbol{y}} \sin K x) .
$$

Using a complex quantity $\bar{M}=M_{x}+i M_{y}$, Eq. (1) can also be written $\bar{M}=M_{0} \mathrm{e}^{i K x} . \hat{\boldsymbol{x}}$ and $\hat{\boldsymbol{y}}$ are unit vectors.

We choose the same parameters as for the design in Ref. [14], thus $L=5.2 \mathrm{~cm}$ and $K=1.21 \mathrm{~cm}^{-1}$. The special feature of the Halbach system is that it generates a magnetic field only on one side of the magnets, namely in the half space $y>0$ for clockwise sense of rotation. This Halbach field is

$$
\mathbf{B}_{H}(x, y)=B_{0} \mathrm{e}^{-K y}(\hat{\boldsymbol{x}} \cos K x-\hat{\boldsymbol{y}} \sin K x),
$$




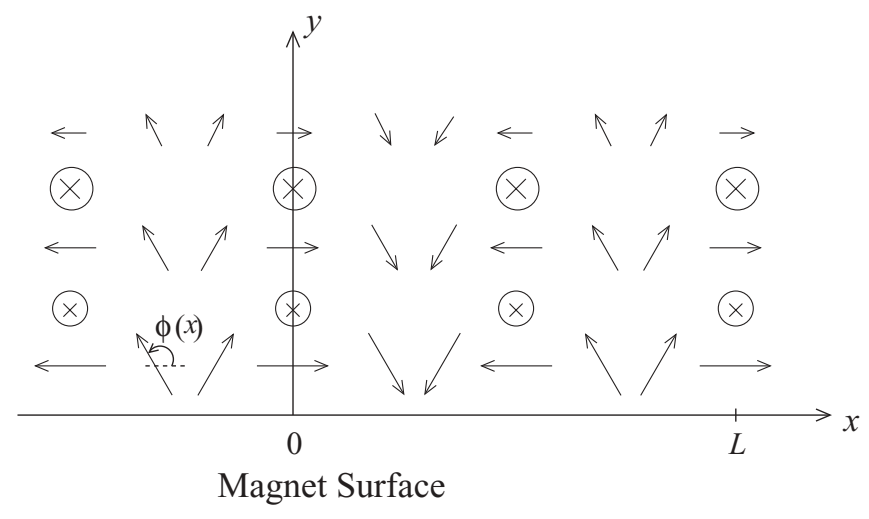

FIG. 1. For our field model, the arrows show the Halbach magnetic field $\boldsymbol{B}_{H}$ as it rotates in the $(x y)$ plane. Its magnitude $B_{H}$ decreases exponentially with height $y$ and is represented by the arrow length using a log scale. The angle $\phi=-K x$ of the Halbach field is also shown. The superimposed stabilization field $\boldsymbol{B}_{1}$ in the $z$ direction increases slowly with $y$ as in Ref. [14] and is symbolized by the crosses of variable size.

or, in complex notation, $\bar{B}_{H}=B_{0} \mathrm{e}^{-K y} \mathrm{e}^{-i K x} . B_{0}=B_{\text {rem }}(1-$ $\left.\mathrm{e}^{-K d}\right)$ is determined by the remanent field $B_{\text {rem }}$ and the block depth $d=2.54 \mathrm{~cm}$. The magnitude of $\mathbf{B}_{H}, B_{H}=B_{0} \mathrm{e}^{-K y}$, only depends on the vertical coordinate $y$. The field distribution is shown schematically in Fig. 1.

In the actual scheme [14], the uniform rotation is replaced by dividing the rotation period $L$ into four blocks, each of length $L / 4$ and with the same magnetization $M$, but with an angle of $90^{\circ}$ between the directions of $\mathbf{M}$ in adjacent blocks (schematically represented as $\cdots \leftarrow \downarrow \rightarrow \uparrow \leftarrow \cdots$ ). Alternative designs are in the form of vertical or horizontal cylinders where the magnets are assembled along the cylindrical surface: in a dipolar way in Ref. [11], with adjacent blocks magnetized in the peripheral direction with equal magnetic poles facing each other (schematically: $\cdots \rightarrow \leftarrow \rightarrow \leftarrow \cdots$ ). In Ref. [12] a cylindrical octupole Halbach magnet is used where 32 blocks are distributed uniformly over the perimeter and the direction of magnetization advances by $56.25^{\circ}$ from one block to the next. In these systems the magnitude $B$ of magnetic field increases sharply near the magnetic wall. A superconducting quadrupole system of magnetic UCN storage for a neutron lifetime experiment is used in Ref. [15] while in Ref. [13] the UCN are stored in superfluid helium using a Ioffe system with horizontal electromagnetic quadrupole. For a quadrupole the field magnitude increases linearly with radial distance from the axis. At least one magnetic end cap is required for all cylindrical systems; on the upper side of vertical systems gravitational confinement can be used.

The field distribution for systems using permanent magnets with magnetization direction advancing in steps from block to block may be expressed as a Fourier series, as in Eq. (7) of Ref. [14]. The first term of the expansion is dominant and has the form (2) with constant $B_{0}$ somewhat smaller than $B_{\text {rem }}\left(1-\mathrm{e}^{-K d}\right)$. For the planar quadrupole Halbach system the reduction factor is $4 /(\pi \sqrt{2})=0.900 \ldots$ [14] and the field in the lower half space $y<-d$ no longer vanishes. The higher Fourier components generate a ripple field in the (xy)-plane, which is significant within $\sim 1 \mathrm{~mm}$ from the magnet surface (and even induces a logarithmic divergence in the field gradient within $\sim 1 \mu \mathrm{m}$ ). But this space is not accessible to the UCNs if we choose a spectrum soft enough to ensure that all neutrons approaching the magnet from above are reflected back up before reaching the ripple zone. We use the value of $0.64 \mathrm{~T}$ for the magnetic field at a safe distance $2 \mathrm{~mm}$, which corresponds to $B_{0}=0.82 \mathrm{~T}$ at the surface. Thus, for the Halbach array generated field we assume the form (2) with $B_{0}=0.82 \mathrm{~T}$ and strict confinement of the vector $\mathbf{B}_{H}$ in the $(x y)$ plane. We ignore the small field ripple in the $z$ direction considered in Ref. [14] since it also decays strongly with distance from the magnet surface. These small perturbations are not expected to affect the depolarization results obtained below in a significant way. Our value of $B_{0}$ is $\sim 20 \%$ lower than the design value of [14] to take into account partial demagnetization, over time, of the $\mathrm{NdFeB}$ permanent magnets exposed to large fields.

A common feature of the various magnetic UCN storage schemes is the requirement of a bias field $\mathbf{B}_{1}$ perpendicular to the main field. It ensures that the field magnitude $B=$ $\left|\mathbf{B}_{H}+\mathbf{B}_{1}\right|$ exceeds a certain minimum value everywhere in the storage volume, especially at critical positions where the field rotates fast in the neutron's moving reference frame. The main purpose of the present work is to provide an estimate of this minimum field for typical field parameters, as those in Ref. [14] where the field $\mathbf{B}_{1}$ also serves the purpose of guiding the decay electrons out of the storage space to a detector as a way to monitor the neutron decay rate in real time. $\mathbf{B}_{1}$ is generated by a toroidal electromagnet, and it is oriented along the longitudinal direction of the "bathtub surface" which corresponds to the $z$ direction in our model with a planar, rather than curved Halbach magnet. We use the same $y$ dependence as in Ref. [14], $\mathbf{B}_{1}=\hat{\mathbf{z}} B_{10} \rho /(\rho-y)$ with $\rho=1.5 \mathrm{~m}$. The magnitude of $\mathbf{B}_{1}, B_{1}=B_{10} \rho /(\rho-y)$ is uniform on a horizontal plane, and since the Halbach field magnitude $B_{H}$ is uniform for given height $y$ no horizontal force acts on the stored UCN.

In Ref. [14] a value of 0.05 to $0.1 \mathrm{~T}$ was proposed for $B_{10}$. We will consider field strengths down to the $\mathrm{mT}$ range since this range seems to be closer to the bias field used in Ref. [11]. In this latter work the value used was not given but it was estimated, on the basis of the Majorana formula, that a minimum field $B_{1}$ of $\sim 0.001 \mathrm{~T}$ was required for their neutron lifetime measurement.

Our analysis of the evolution of spin-flip probability for UCNs moving in our model magnetic field distribution differs from that of Ref. [14] as follows. It is not restricted to purely vertical motion but assumes that the UCNs can also have arbitrary horizontal velocity components $v_{x}$ and $v_{z} . v_{x}$ and $v_{z}$ are constant since neither gravity nor the net magnetic field $\mathbf{B}=\mathbf{B}_{H}+\mathbf{B}_{1}$ of our model exert a horizontal force on the neutron. As noted earlier, both $B_{H}$ and $B_{1}$ are uniform at given height $y$, and $\mathbf{B}_{H}$ is perpendicular to $\mathbf{B}_{1}$, thus $B=\sqrt{B_{H}^{2}+B_{1}^{2}}$ depends on $y$ only. As a result, the magnetic force, which is determined by the gradient of $\mathbf{B}$, has no horizontal components and the equation of motion is separable in three dimensions.

We use three different methods of analysis, both for purely magnetic confinement of UCNs with arbitrary 3D velocity components, and for a system involving a nonmagnetic mirror 
placed into our model magnetic field distribution: (a) in Sec. III a quantum approach using the WKB approximation, (b) in Sec. IV a quasiclassical approximation, and (c) in Sec. V direct numerical integration of the equations of motion. In the quantum approach the stationary spin-dependent Schrödinger equation is solved using the WKB approximation, extending the method of Ref. [14] to 3D motion. The semiclassical Majorana method [18] which was adapted to magnetically confined UCNs in Ref. [14] will also be extended to 3D. Using both methods we will also analyze UCN reflection on a nonmagnetic mirror in a magnetic field. In Sec. V we will show that some analytic results can be obtained as well by direct numerical integration of the wave equation for the spin-flipped component of the wave function. This is only feasible because the magnetically trapped UCNs have relatively long wavelengths, in the $\mu \mathrm{m}$ range, so the number of wave oscillations for the entire integration path is not too large.

\section{QUANTUM MECHANICAL APPROACH}

\section{A. Basic equations}

The wave function for a UCN moving in the magnetogravitational field of the trap is a linear superposition of the two eigenstates of the magnetic moment interaction Hamiltonian

$$
\mathcal{H}_{m}=-\mu_{\mathrm{n}} \boldsymbol{\sigma} \cdot \mathbf{B}
$$

where $\mu_{\mathrm{n}}=-1.913 \mu_{\mathrm{N}}$ is the neutron magnetic moment in terms of the nuclear magneton $\mu_{\mathrm{N}}=0.505 \times 10^{-26} \mathrm{~J} / \mathrm{T}, \sigma$ is the Pauli spin operator, and $\boldsymbol{B}$ is the local magnetic field. The two eigenstates $\chi^{+}$and $\chi^{-}$of $\mathcal{H}_{m}$ satisfy the eigenvalue equations

$$
\mathcal{H}_{m} \chi^{ \pm}= \pm\left|\mu_{\mathrm{n}}\right| B \chi^{ \pm}
$$

and correspond, respectively, to neutron spin parallel to $\mathbf{B}$ with spin energy $+\left|\mu_{\mathrm{n}}\right| B$, and to antiparallel spin with energy $-\left|\mu_{\mathrm{n}}\right| B$. These spin eigenfunctions are obtained by spin rotation from the $z$ axis to the direction of $\boldsymbol{B}$ through angles $\theta$ and $\phi$. The polar field angle is $\theta=\cos ^{-1}\left(B_{z} / B\right)=\sin ^{-1}\left(B_{x y} / B\right)$, where $B_{z}=B_{1}$ is due to the bias field $\boldsymbol{B}_{1}$ and $B_{x y}=B_{H}$ is the magnitude of the Halbach field $\mathbf{B}_{H}$. The azimuthal angle in the (xy) plane is $\phi=\sin ^{-1}\left(B_{y} / B_{x y}\right)=\tan ^{-1}\left(B_{y} / B_{x}\right)$.

Equation (2) shows that for the Halbach field configuration

$$
\phi=-K x .
$$

Thus $\phi$ depends only on $x$ (not on $y$ or $z$ ), while $\theta$ depends only on $y$. These properties will simplify the analysis considerably. Exact correspondence between the system of coordinates $x, y, z$ used here and the system $\eta, \zeta, \xi$ used in Ref. [14] (with $\zeta$ pointing up) is established if we add the constant $\pi / 2$ to the right-hand side of Eq. (5).

Performing the spin rotation through angles $\theta$ and $\phi$ we obtain for the spin basis vectors [22] with quantization axis along $-\mathbf{B}$

$$
\chi^{+}=\left(\begin{array}{c}
e_{-} s \\
-c
\end{array}\right), \quad \chi^{-}=\left(\begin{array}{c}
c \\
e_{+} s
\end{array}\right),
$$

where $s=\sin (\theta / 2), c=\cos (\theta / 2)$, and $e_{ \pm}=\exp ( \pm i \phi)=$ $\exp (\mp i K x)$. We chose the basis vectors in the form (6) to establish formal correspondence with the basis vectors used in Ref. [14]. This entails a change of sign for $\theta, \phi$ and $K$ in intermediary results for wave amplitudes, affecting only their phases but not the final results for depolarization probabilities. We write the dependence of the wave function on position and spin in the form

$$
\chi=\alpha^{(3)}(x, y, z) \chi^{+}+\beta^{(3)}(x, y, z) \chi^{-},
$$

where we have used the superscript (3) to indicate that $\alpha^{(3)}(x, y, z), \beta^{(3)}(x, y, z)$ are functions of the three space coordinates while the corresponding functions $\alpha(y)$ and $\beta(y)$, introduced below, depend on $y$ only. $\chi$ satisfies the eigenvalue equation

$$
E \chi=\left[-\frac{\hbar^{2}}{2 m} \nabla^{2}+m g y+\left|\mu_{\mathrm{n}}\right| \boldsymbol{\sigma} \cdot \boldsymbol{B}\right] \chi
$$

for a neutron of mass $m$ with constant total energy $E$ moving in a uniform gravitational field of magnitude $g$ and a nonuniform magnetic field $\boldsymbol{B}$. Using subscripts to denote partial differentiation, the Laplace operator acting on the wave function gives

$$
\begin{aligned}
\nabla^{2} \chi= & \left(\alpha_{x x}^{(3)} \chi^{+}+2 \alpha_{x}^{(3)} \chi_{x}^{+}+\alpha^{(3)} \chi_{x x}^{+}+\beta_{x x}^{(3)} \chi^{-}\right. \\
& \left.+2 \beta_{x}^{(3)} \chi_{x}^{-}+\beta^{(3)} \chi_{x x}^{-}\right)+(x \rightarrow y)+(y \rightarrow z),
\end{aligned}
$$

where for the second and third term the indicated permutations are performed. The basis vectors for quantization along the fixed $z$ axis can be expressed in terms of the basis vectors $\chi^{+}$ and $\chi^{-}$:

$$
\left(\begin{array}{l}
1 \\
0
\end{array}\right)=s e_{+} \chi^{+}+c \chi^{-}, \quad\left(\begin{array}{l}
0 \\
1
\end{array}\right)=-c \chi^{+}+s e_{-} \chi^{-} .
$$

Using Eq. (10) and noting that, from Eq. (5), $\phi_{x}=-K$, $\phi_{x x}=0, \phi_{y}=\phi_{z}=0$ and also $\theta_{x}=\theta_{z}=0$, we obtain

$$
\begin{aligned}
& \chi_{x}^{+}=i s K\left(s \chi^{+}+c e_{-} \chi^{-}\right), \quad \chi_{x}^{-}=i s K\left(c e_{+} \chi^{+}-s \chi^{-}\right), \\
& \chi_{x x}^{+}=i K \chi_{x}^{+}, \quad \chi_{x x}^{-}=-i K \chi_{x}^{-}, \quad \chi_{y}^{+}=\frac{1}{2} e_{-} \theta_{y} \chi^{-}, \\
& \chi_{y}^{-}=-\frac{1}{2} e_{+} \theta_{y} \chi^{+}, \quad \chi_{y y}^{+}=\frac{1}{2}\left(\theta_{y y} e_{-} \chi^{-}-\frac{1}{2} \theta_{y}^{2} \chi^{+}\right), \quad(11) \\
& \chi_{y y}^{-}=-\frac{1}{2}\left(\frac{1}{2} \theta_{y}^{2} \chi^{-}+\theta_{y y} e_{+} \chi^{+}\right), \\
& \chi_{z}^{ \pm}=0, \quad \chi_{z z}^{ \pm}=0 .
\end{aligned}
$$

As in Ref. [14] we will use the WKB approximation [23] and keep only those terms in Eq. (9) that contain the derivatives of the field variables $(\theta$ and $\phi)$ in lowest order since those change on the scale of centimeters while the waves in real space, $\alpha^{(3)}$ and $\beta^{(3)}$, vary on the micrometer scale, i.e., $\sim 10^{4}$ times faster. This implies that all second derivatives of $\chi^{+}$ and $\chi^{-}$are dropped, along with other small terms. (Using the numerical integration described in Sec. V we have performed test runs where the small terms were retained. The results were the same within the precision of numerical integration.) Assuming that the UCN started out from a pure (+) spin state and keeping only the dominant terms in Eq. (9) we obtain

$$
\begin{aligned}
\nabla^{2} \chi= & \left(\alpha_{x x}^{(3)}+\alpha_{y y}^{(3)}+\alpha_{z z}^{(3)}\right) \chi^{+}+\left[\beta_{x x}^{(3)}+\beta_{y y}^{(3)}+\beta_{z z}^{(3)}\right. \\
& \left.+\mathrm{e}^{i K x}\left(\theta_{y} \alpha_{y}^{(3)}+i K \alpha_{x}^{(3)} \sin \theta\right)\right] \chi^{-}
\end{aligned}
$$

where we have used $\sin \theta=2 s c, \phi=-K x$, and the fact that in practice $\left|\beta^{(3)}\right| \ll\left|\alpha^{(3)}\right|$. The functions in real space multiplying $\chi^{+}$and $\chi^{-}$can be simplified by noting that the 
$x$ and $z$ dependence of $\alpha^{(3)}$ has the plane wave form $\mathrm{e}^{i k_{x} x} \mathrm{e}^{i k_{z} z}$ and $\beta^{(3)}$ is proportional to $\mathrm{e}^{i K x} \mathrm{e}^{i k_{x} x} \mathrm{e}^{i k_{z} z}$. The wave numbers $k_{x}$ and $k_{z}$ are constant and $\mathrm{e}^{i K x}$ represents a Bloch-wave modulation due to the periodicity of the Halbach field. In practice, $k_{x}$ and $k_{z}$ are of order $\mu \mathrm{m}^{-1}$, thus much larger than $K$ and $\theta_{y}$, both of which are of order $\mathrm{cm}^{-1}$.

Thus we can factor Eq. (12) in the form

$$
\begin{aligned}
\nabla^{2} \chi= & \mathrm{e}^{i k_{x} x} \mathrm{e}^{i k_{z} z}\left\{\left[\alpha^{\prime \prime}-\left(k_{x}^{2}+k_{z}^{2}\right) \alpha\right] \chi^{+}+\mathrm{e}^{i K x}\left[\beta^{\prime \prime}\right.\right. \\
& \left.\left.-\left(k_{x}^{2}+k_{z}^{2}\right) \beta+\left(\theta^{\prime} \alpha^{\prime}-K k_{x} \alpha \sin \theta\right)\right] \chi^{-}\right\},
\end{aligned}
$$

simplifying the notation. In Eq. (13) and henceforth, $\alpha(y)$ and $\beta(y)$ stand for the $y$-dependent parts of the wave function only, and differentiation with respect to $y$ is denoted by primes. We also drop the subscript $y$ from the $y$-component of the wave vector. Thus, $\alpha^{(3)}(x, y, z)=\alpha(y) \mathrm{e}^{i k_{x} x} \mathrm{e}^{i k_{z} z}$ and $\beta^{(3)}(x, y, z)=$ $\beta(y) \mathrm{e}^{i K x} \mathrm{e}^{i k_{x} x} \mathrm{e}^{i k_{z} z}$. The terminology $\alpha(y), \beta(y)$ conforms to that used in Ref. [14] where motion in horizontal directions was not taken into account in the depolarization calculations.

Inserting Eq. (13) into the eigenvalue equation (8) gives [14] two coupled equations, one for spinor $\chi^{+}$(i.e., for low-field seeking UCNs with spin parallel to $\boldsymbol{B}$, which can be stored) and the other for $\chi^{-}$(i.e., for the fraction of UCNs whose spin has flipped relative to $\boldsymbol{B}$ and which therefore can escape from the trap; the probability of flipping twice is negligible):

$$
E \alpha=-\frac{\hbar^{2}}{2 m}\left[\alpha^{\prime \prime}-\left(k_{x}^{2}+k_{z}^{2}\right) \alpha\right]+m g y \alpha+\left|\mu_{\mathrm{n}}\right| B \alpha
$$

and

$$
\begin{aligned}
E \beta= & -\frac{\hbar^{2}}{2 m}\left[\beta^{\prime \prime}-\left(k_{x}^{2}+k_{z}^{2}\right) \beta+\left(\theta^{\prime} \alpha^{\prime}-K k_{x} \alpha \sin \theta\right)\right] \\
& +m g y \beta-\left|\mu_{n}\right| B \beta .
\end{aligned}
$$

In the framework of the WKB approximation, the solution of Eq. (14) is [14]

$$
\alpha(y)=k_{+}^{-1 / 2}(y) \exp \left( \pm i \Phi_{+}(y)\right),
$$

where

$$
\frac{\hbar^{2} k_{ \pm}^{2}(y)}{2 m}=E-\frac{\hbar^{2}}{2 m}\left(k_{x}^{2}+k_{z}^{2}\right)+m g\left(y_{0}-y\right) \mp\left|\mu_{\mathrm{n}}\right| B(y) .
$$

$k_{+}(y)$ is the magnitude of the $y$ component of local wave vector for the storable (+) spin state (parallel to B) and $k_{-}(y)$ is that for the $(-)$ spin state.

In Eq. (17), $y_{0}$ is the greatest height a neutron of energy $E$ and given $k_{x}$ and $k_{z}$ would achieve in the gravitational field if the magnetic field were switched off. In Eq. (16),

$$
\Phi_{+}(y)=\int_{y_{s}}^{y} k_{+}(u) d u
$$

is the phase angle, for the + spin state, accumulated between the start of vertical motion and the position $y$. The initial height $y_{s}$ for motion upward is assumed to be that of the lower turning point, thus $y_{s^{+}}=y_{l}$, and for motion downward the initial level is taken at the upper turning point, $y_{s^{-}}=y_{u}$. The additional + or - sign in the argument of the exponential function in Eq. (16), in front of $\Phi_{+}$, refers to this direction of the motion; plus for upward and minus for downward, as in [14].
The WKB wave function (16) is normalized to a constant particle flux $\hbar / m$ in the $y$ direction. For the spin-flipped UCNs, the flux in the $y$ direction is the measure of the probability of depolarization, as shown below. At the classical turning points, where $k_{+}=0$, the WKB form (16) diverges and has to be replaced by the Airy function, as shown in Ref. [14], but the WKB form is still valid almost all the way to the turning point, except for the last $\mu \mathrm{m}$ or so, since it correctly represents the asymptotic behavior of the Airy function in this region.

This is an important feature of the approximation used in Ref. [14] and in the present work. It is made more explicit as follows: The asymptotic form of the Airy function in the region of real waves (rather than the exponentially decaying wave on the other side) is [14,24]

$$
\begin{aligned}
\operatorname{Ai}\left(-a_{s}\left|y-y_{s}\right|\right) \sim & \left(\frac{a_{s}}{\pi k_{s}}\right)^{1 / 2} \cos \left(k_{s}\left|y-y_{s}\right|-\frac{i \pi}{4}\right) \\
= & \frac{1}{2}\left(\frac{a_{s}}{\pi k_{s}}\right)^{1 / 2}\left\{\exp \left(i k_{s}\left|y-y_{s}\right|-\frac{i \pi}{4}\right)\right. \\
& \left.+\exp \left(-i k_{s}\left|y-y_{s}\right|+\frac{i \pi}{4}\right)\right\} .
\end{aligned}
$$

The wave number $k_{s}=\frac{m}{\hbar}\left(2 g_{+s}\right)^{1 / 2}\left|y-y_{s}\right|^{1 / 2}$ is determined by the local acceleration $g_{+s}=\left|g+\left(\frac{\left|\mu_{\mathrm{n}}\right|}{m}\right)\left(\frac{d B}{d y}\right)_{s}\right|$ at the turning point $y_{s}$, and $a_{s}=\left(\frac{m}{\hbar}\right)^{2 / 3}\left(2 g_{+s}\right)^{1 / 3}$. For upward motion the WKB wave approximation (16) for $\alpha(y)$, which is valid between the turning points, is matched to the first term inside the braces of Eq. (19), and for downward motion to the second term by adjusting the constant multiplying the Airy function.

It follows from Eq. (15) that the wave function $\beta(x, y)$ for the spin flipped component is determined by the inhomogeneous second-order differential equation

$$
\beta^{\prime \prime}(y)+k_{-}^{2}(y) \beta(y)=-\theta^{\prime}(y) \alpha^{\prime}(y)+K k_{x} \alpha(y) \sin \theta(y) .
$$

Having separated off the $x$ and $z$ dependences allows us to choose the same WKB form for $\beta(y)$ as in Ref. [14]:

$$
\beta(y)=k_{-}^{-1 / 2}(y) \exp \left( \pm i \Phi_{-}(y)\right) f(y),
$$

where the function $f(y)$ modulating the WKB wave represents the amplitude of spin flip. Apart from the modulation $f(y)$, $\beta(y)$ is constructed in the same way as $\alpha(y)$. The phase accumulated since the start at a turning point,

$$
\Phi_{-}(y)=\int_{y_{s}}^{y} k_{-}(u) d u,
$$

always has a larger magnitude than the phase $\Phi_{+}(y)$ for $\alpha(y)$ since $k_{-}$is greater than $k_{+}$(except in zero magnetic field).

Summarizing, the governing equation for $\beta(y)$ is the second-order differential equation

$$
\begin{aligned}
& \beta^{\prime \prime}(y)+k_{-}^{2}(y) \beta(y) \\
& \quad=-\theta^{\prime}(y) \alpha^{\prime}(y)+K k_{x} \alpha(y) \sin \theta(y) \\
& \quad=-\left[ \pm i k_{+} \theta^{\prime}(y)-K k_{x} \sin \theta(y)\right] \alpha(y),
\end{aligned}
$$

where the right-hand side represents an inhomogeneous term and $\alpha(y)$ is given by Eq. (16) while $\beta(y)$ has the form

$$
\beta(y)=k_{-}^{-1 / 2}(y) \exp \left( \pm i \Phi_{-}(y)\right) f(y) .
$$


In the second expression on the right-hand side of Eq. (23) we have carried out the differentiation of $\alpha(y)$, using the WKB rule of considering the slow-varying terms as constant, with the result $\alpha^{\prime}= \pm i k_{+} \alpha(y)$ where the "+ " sign applies to upward motion and the "-" sign to downward motion. This replacement is valid except within a few $\mu \mathrm{m}$ of the turning points.

Our Eq. (23) is the same as Eq. (28) of Ref. [14] except for the additional, $k_{x}$ dependent term on the right-hand side. It is present because we include motion with finite lateral momentum $\hbar k_{x}$, whereas the analysis in Ref. [14] was restricted to the special case $k_{x}=0$. We will show that this new term makes the major contribution to UCN depolarization in magnetic storage. We also note that Eq. (23) does not depend on $k_{z}$, thus motion exactly along the $z$ direction does not induce depolarization. This is understandable since neutrons moving along the $z$ axis during the short periods of horizontal motion at the turning points move in a uniform B-field. On the other hand, in motion along the $x$ direction they are exposed to the strong field ripple due to the rotating Halbach field. These features are expected to hold, on a qualitative basis, also for the "bathtub system" of Ref. [14], where the direction perpendicular (parallel) to the curved Halbach array corresponds to our $z$ axis ( $x$ axis).

Next we will solve Eq. (23) to obtain the depolarization rate for UCN storage in our model field distribution.

\section{B. Depolarization in magnetic storage}

\section{Mathematical approach}

We will first consider a neutron of given energy and fixed values of $k_{x}$ and $k_{z}$, moving downward from the upper turning point $y_{u}$. Using the WKB rule, the second derivative $\beta^{\prime \prime}$ is obtained from Eq. (24) as

$$
\beta^{\prime \prime}(y)=k_{-}^{-1 / 2}(y) \exp \left(-i \Phi_{-}(y)\right)\left(f^{\prime \prime}-2 i k_{-} f^{\prime}-k_{-}^{2} f\right) .
$$

Inserting $\beta^{\prime \prime}(y)$ and $\alpha(y)$ from Eq. (16) into Eq. (23) gives

$$
f^{\prime \prime}-2 i k_{-} f^{\prime}=[i U(y)+V(y)] \exp (-i \Phi(y)),
$$

where $\Phi=\Phi_{+}-\Phi_{-}, U(y)=\left(k_{+} k_{-}\right)^{1 / 2} \theta^{\prime}$, and $V(y)=$ $\left(\frac{k_{-}}{k_{+}}\right)^{1 / 2} K k_{x} \sin \theta$.

Defining a function $F(y)$ through $f^{\prime}=F \exp \left(2 i \Phi_{-}\right)$we obtain from Eq. (26)

$$
F^{\prime}(y)=[i U(y)+V(y)] \exp (-i \Psi(y)),
$$

where $\Psi=\Phi_{+}+\Phi_{-}$. The phases $\Phi_{+}, \Phi_{-}, \Phi$ and $\Psi$ are fastvarying quantities, while the field variables $U$ and $V$ vary slowly with $y$ and will therefore be considered constant in all differentiations. In integrations, as needed to obtain $F(y)$ from Eq. (27), this "WKB rule" directly corresponds to performing the integral of products of slow- and fast-varying terms by parts and neglecting the second term which contains the derivative of the slow-varying factor. It has been shown numerically in Ref. [14] that for the parameters of magnetic UCN storage at hand this procedure gives approximations with precision in the range $10^{-4}$. Thus we obtain from Eq. (27)

$$
\begin{aligned}
F(y) & =\int_{y_{s}}^{y}\left[i U\left(y^{\prime}\right)+V\left(y^{\prime}\right)\right] \exp \left(-i \Psi^{\prime}\right) d y^{\prime} \\
& =\int_{\Psi_{s}}^{\Psi} \frac{i U\left(y^{\prime}\right)+V\left(y^{\prime}\right)}{k_{-}+k_{+}} \exp \left(-i \Psi^{\prime}\right) d \Psi^{\prime} \\
& =\frac{i[i U(y)+V(y)]}{k_{-}(y)+k_{+}(y)} \exp (-i \Psi(y)) .
\end{aligned}
$$

The third step in Eq. (28) is an integration by parts, where only the leading term is kept.

The lower limit of the $y$ integration in Eq. (28) is the upper turning point. Carrying out the integration in the last step of Eq. (28) we should expect a contribution from this lower limit of integration ( $y_{s} \approx y_{u}$ or $\Psi_{s} \approx 0$ ). However, such a term does not appear in Eq. (28) for the following reasons: First we note that in the quantum treatment involving the Airy function the turning "point" is blurred within a range of order $\mu \mathrm{m}$. Second, as mentioned in Sec. I, we assume, as the authors of Ref. [14] did, that at a turning "point" (here the region around $y_{u}$ ) the neutron starts out in a pure low-field seeking spin state $(+)$, i.e., from $\beta=0, f=0$. This implies that $\alpha(y)$ and the functions $\beta(y), f(y)$ and $F(y)$, derived from $\alpha$ and $\alpha^{\prime}$ through Eq. (23), tend to 0 as $y \rightarrow+\infty$ (in practice, for $y$ just a few $\mu \mathrm{m}$ above the classical turning point). It was also mentioned earlier, that the WKB function used here for $\alpha(y)$ is just the asymptotic representation of the Airy function $\mathrm{Ai}$ which does satisfy the initial condition without any constant added since $\mathrm{Ai}$ and $\mathrm{Ai}^{\prime}$ vanish for $y \rightarrow+\infty$. As a result, there is no lower-limit contribution to $F$ in Eq. (28), and the same is true also for the functions $f(y)$ and $\beta(y)$ derived below by further integration [in Eqs. (29) and (32)]. We can also argue that, due to the factor $k_{+}^{-1 / 2}(y)$ in $V(y)$ [defined following Eq. (26)], a wave containing a term derived from a finite integration constant in Eq. (28) would diverge at the endpoint $y_{l}$ of integration, where $k_{+}=0$, and therefore must be zero, in the same way as in total reflection a wave increasing exponentially inside the medium must have amplitude zero. The singularity is avoided only by setting the integration constant in Eq. (28) equal to zero.

Remembering the definition $F=f^{\prime} \exp \left(-2 i \Phi_{-}\right)$, we integrate Eq. (28) once more to obtain

$$
\begin{aligned}
f(y) & =\int_{y_{s}}^{y} F\left(y^{\prime}\right) \exp \left(2 i \Phi_{-}^{\prime}\right) d y^{\prime} \\
& =i \int_{y_{s}}^{y} \frac{i U\left(y^{\prime}\right)+V\left(y^{\prime}\right)}{k_{-}+k_{+}} \exp \left(-i \Phi^{\prime}\right) d y^{\prime} \\
& =-i \int_{\Phi_{s}}^{\Phi} \frac{i U\left(y^{\prime}\right)+V\left(y^{\prime}\right)}{k_{-}^{2}-k_{+}^{2}} \exp \left(-i \Phi^{\prime}\right) d \Phi^{\prime} \\
& =P(y) \exp (-i \Phi) .
\end{aligned}
$$

In Eq. (29), we have defined

$$
\begin{aligned}
P(y) & =\frac{i U(y)+V(y)}{W(y)}, \\
W(y) & =k_{-}^{2}(y)-k_{+}^{2}(y) .
\end{aligned}
$$


It follows from the definition of $k_{+}$and $k_{-}$in Eq. (17) that

$$
W=k_{-}^{2}-k_{+}^{2}=\frac{4 m}{\hbar^{2}}\left|\mu_{\mathrm{n}}\right| B(y)
$$

depends only on the magnitude $B(y)$ of the local magnetic field.

From the symmetry of the problem it follows that motion in the opposite direction, from the lower turning point at $y_{l}$ upward to $y_{u}$ gives the same function $f(y)$ as in the last form of Eq. (29), except that the phase term $\exp (-i \Phi)$ is replaced by $\xi \exp (+i \Phi)$ and $P(y)$ is replaced by its complex conjugate $P^{*}(y)$. $\xi$ is a phase factor of unit amplitude, which arises due to the shift of reference point for $\Phi$ from $y_{u}$ to $y_{l}$ when we change from downward to upward motion. None of these differences affect the squared magnitude $|f(y)|^{2}$ which measures the probability to find the neutron in the spin-flipped state at height $y$. An explicit expression for $\xi$ will be given following Eq. (44).

We can compare our Eq. (29) for the depolarization amplitude $f$ with the corresponding result in Eqs. (31)-(35) of Ref. [14], where only the case $k_{x}=0$ was analyzed. This corresponds to setting $V(y)=0$ in our analysis. Apart from this difference, our Eq. (29) can be obtained from Eq. (35) of [14] by multiplying the latter by the factor $2 i k_{+} /\left(k_{-}+k_{+}\right)$. The magnitude of this factor is close to unity if $k_{+}(y)$ is only slightly less than $k_{-}(y)$. For fairly high-energy UCNs this is the case for most of the path between the turning points, but not near these points. This minor difference appears to be due to the neglect, in Ref. [14], of $f^{\prime \prime}$ in the derivation of their Eq. (31).

The main difference between the results of [14] and our numbers, obtained below, is due to the restriction of the previous work to $k_{x}=0$. Our analysis of the depolarization current and depolarization rate for magnetically stored UCNs yields a loss $\sim 10$ decades larger for a typical velocity $v_{x}$ up to $\pm 3 \mathrm{~m} / \mathrm{s}$ than the range of values, $10^{-20}$ to $10^{-23}$ given in Ref. [14] for $v_{x}=0$.

\section{Interpretation in terms of loss current and depolarization rate}

We can now insert $f(y)$ from Eq. (29) into Eq. (24) to determine the wave function $\beta(y)$ for spin-flipped UCNs. For the downward motion, this gives

$$
\beta(y)=k_{-}^{-1 / 2}(y) P(y) \exp \left(-i \Phi_{+}(y)\right) .
$$

The phase $\Phi_{+}$(with the index + ) indicates that this wave for the $(-)$ spin state propagates, not with wave number $k_{-}$, but with the same wave number $k_{+}$as the $(+)$spin state, as it should. Using $\beta^{\prime \prime}(y)=-k_{+}^{2}(y) \beta(y)$ we can verify that the function (32) solves Eq. (23), and thus the Schrödinger equation starting from a pure $(+)$ spin state, as described following Eq. (28).

On a more formal basis, Eq. (32) represents a particular solution to Eq. (23) and we could add to Eq. (32) any solution $\beta_{h \pm}(y)$ of the homogeneous equation $\beta_{h}^{\prime \prime}(y)+k_{-}^{2}(y) \beta_{h}(y)=0$ corresponding to Eq. (23). In the WKB framework, these solutions are $\beta_{h \pm}(y)=C_{ \pm} k_{-}^{-1 / 2}(y) \exp \left( \pm i \Phi_{-}(y)\right)$ with arbitrary constants $C_{ \pm}$. These functions represent a constant current in the upward (downward) direction for the $+(-)$ sign. Thus the same current enters and leaves the storage space, resulting in a zero contribution to the net flux out which corresponds to the depolarization loss as described below. As an example, such a homogeneous term may represent a neutron that has undergone a spin flip on the way up, proceeds past $y_{u}$ until it reaches its reversal point in the gravitational field and, on its way down, traverses the storage space without contributing to further depolarization.

Reverting to solution (32) without added terms, we associate the net depolarization over the path from upper turning point $y_{u}$ to $y_{l}$ with the current of spin-flipped UCNs at the endpoint $y_{l}$, which is consistent with the interpretation in Ref. [14]. This current represents the net flux out of the storage space since no flux enters at $y_{u}$.

At an arbitrary position $y$ along the way the current $j_{-}(y)$ is given by [22]

$$
j_{-}(y)=\frac{\hbar}{m} \operatorname{Re}\left[i \beta^{*}(y)\left(\frac{d \beta}{d y}\right)\right] .
$$

For function (32) we have

$$
\begin{aligned}
\beta^{*}(y) & =k_{-}^{-1 / 2}(y) P^{*}(y) \exp \left(i \Phi_{+}(y)\right), \\
\frac{d \beta}{d y} & =-i k_{+}(y) k_{-}^{-1 / 2}(y) P(y) \exp \left(-i \Phi_{+}(y)\right),
\end{aligned}
$$

and thus the current as a function of position $y$ between $y_{l}$ and $y_{u}$ becomes

$$
j_{-}(y)=\frac{\hbar}{m}\left(\frac{k_{+}}{k_{-}}\right)|P|^{2}=\frac{\hbar}{m} \frac{k_{+}^{2} \theta^{\prime 2}+K^{2} k_{x}^{2} \sin ^{2} \theta}{\left(k_{-}^{2}-k_{+}^{2}\right)^{2}} .
$$

The function $(m / \hbar) j_{-}(y)$ corresponds to the depolarization probability of Ref. [14]. It is plotted in Fig. 2 for UCNs with energy for vertical motion determined by the "drop heights"

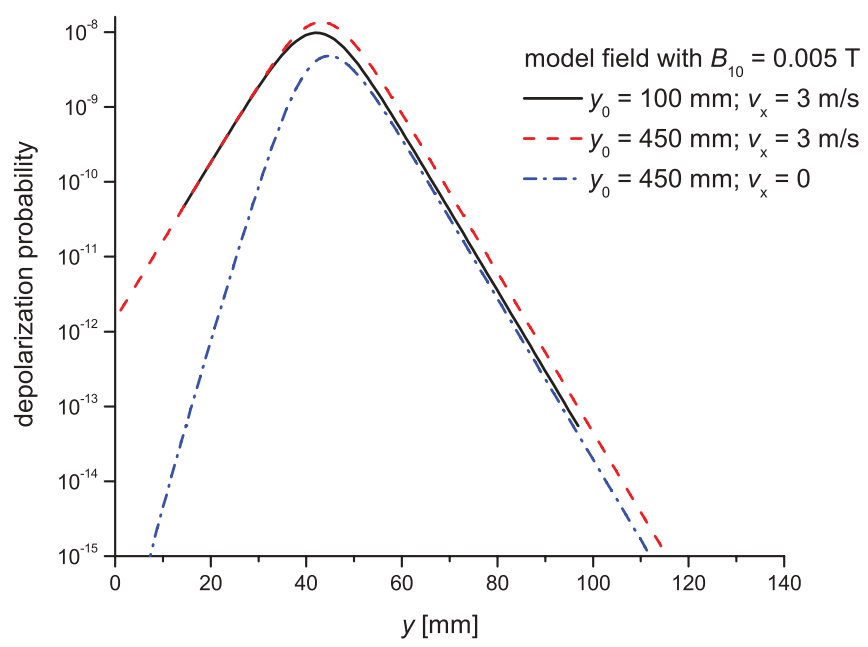

FIG. 2. (Color online) Depolarization probability, given by Eq. (34) multiplied by $m / \hbar$, as a function of neutron position for drop heights $y_{0}=450 \mathrm{~mm}$ and $100 \mathrm{~mm}$, stabilization field parameter $B_{10}=0.005 \mathrm{~T}$, and neutron velocity component $v_{x}=3 \mathrm{~m} / \mathrm{s}$ or zero. The sharp peak occurs in the region where the gradient of field angle $\theta$ is largest. 
$y_{0}=10 \mathrm{~cm}$ and $45 \mathrm{~cm}$ [ $y_{0}$ was defined following Eq. (17)], a bias magnetic field $B_{10}=0.005 \mathrm{~T}$ and $v_{x}=3 \mathrm{~m} / \mathrm{s}$. As in Fig. 3 of Ref. [14] we see a sharp peak at the $y$ position where $\theta^{\prime}$ is large, and a decrease as the particle drops further down. The third curve in Fig. 2 is for $y_{0}=45 \mathrm{~cm}, B_{10}=0.005 \mathrm{~T}$, and $v_{x}=0$. The peak value and the decrease on the upper side are quite similar. Below the peak position the curve for $v_{x}=$ 0 decreases faster than for $v_{x}=3 \mathrm{~m} / \mathrm{s}$.

The current leaving the storage space at $y=y_{l}$ is

$$
\begin{aligned}
j_{l} & =\frac{\hbar}{m}\left(\frac{k_{+l}}{k_{-l}}\right)\left|P_{l}\right|^{2}=\frac{\hbar}{m} \frac{k_{+l}^{2} \theta_{l}^{\prime 2}+K^{2} k_{x}^{2} \sin ^{2} \theta_{l}}{\left(k_{-l}^{2}-k_{+l}^{2}\right)^{2}} \\
& =\frac{\hbar}{m} \frac{K^{2} k_{x}^{2}}{k_{-l}^{4}} \sin ^{2} \theta_{l},
\end{aligned}
$$

where the index $l$ refers to the values at $y=y_{l}$ and the last form of Eq. (35) uses the fact that $k_{+}$vanishes at the turning points. In the analysis of Ref. [14] for $k_{x}=0$ only this vanishing term $\left(\sim k_{+l}^{2} \theta_{l}^{\prime 2}\right)$ appeared, and a higher order of approximation as well as numerical integration were used to estimate the depolarization probability. The result was a very small value which is negligible compared to the second term given in the last form of Eq. (35), even for values of $v_{x}$ as small as $0.1 \mathrm{~m} / \mathrm{s}$.

The dependence of Eq. (35) on the primary field variables is established by noting that $\sin ^{2} \theta=B_{H}^{2} / B^{2}, k_{-l}^{4} \sim B_{l}^{2}$ and $K^{2} k_{x}^{2}=\left(\frac{m}{\hbar}\right)^{2} \omega^{2}$, where $\omega=2 \pi v_{x} / L$ is the frequency of the Halbach field as seen by the moving UCN.

For upward motion from $y_{l}$ to $y_{u}$ we get the same result for the current as in Eq. (35) except that all indices $l$ are replaced by $u$, i.e., the quantities relevant for the spin-flipped current leaving the system at the upper turning point are determined by the field angle $\theta_{u}$ and by $k_{-u}$ at $y_{u}$.

The combined depolarization loss for one reflection on the magnetic field, i.e., for one complete round trip down and up, is determined by

$$
\frac{m}{\hbar}\left(j_{l}+j_{u}\right)=K^{2} k_{x}^{2}\left(\frac{\sin ^{2} \theta_{l}}{k_{-l}^{4}}+\frac{\sin ^{2} \theta_{u}}{k_{-u}^{4}}\right) .
$$

To approximate the actual situation in magnetic UCN storage, where the UCNs have positive and negative velocities in any direction and, for a low-energy Maxwell spectrum, with uniform probability per unit of $k_{x}, k_{y}$, and $k_{z}$ (since the phase space density is constant), we take the mean value of $k_{x}^{2}$ in Eq. (36) for the spectral interval $-k_{x, \max }<k_{x}<+k_{x \text {, max }}$ with the result

$$
\frac{m}{\hbar}\left\langle j_{l}+j_{u}\right\rangle=K^{2}\left(\frac{k_{x, \max }^{2}}{3}\right)\left(\frac{\sin ^{2} \theta_{l}}{k_{-l}^{4}}+\frac{\sin ^{2} \theta_{u}}{k_{-u}^{4}}\right) .
$$

As a final step in this analysis of depolarization in the WKB approximation we establish the explicit connection between the loss current (37) and the rate of depolarization, $\tau_{\mathrm{dep}}^{-1}$, that is observable as a contribution to the decay rate (but should be negligible compared to the neutron $\beta$-decay rate in a neutron lifetime measurement). For given neutron energy for vertical motion, i.e., fixed turning levels at $y_{l}$ and $y_{u}$, the depolarization rate (in $\mathrm{s}^{-1}$ ) is determined by the loss current (37) divided by the number of UCNs in the field-repelled spin state present in the trap,

$$
N=2 \int_{y_{l}}^{y_{u}}|\alpha(y)|^{2} d y=2 \int_{y_{l}}^{y_{u}} \frac{1}{k_{+}(y)} d y .
$$

We have used the square magnitude of the WKB form (16) for $\alpha(y)$ as the density. The factor 2 takes into account that both downward and upward moving UCNs are in the trap at the same time.

Since $k_{+}=(m / \hbar) v_{+}$and $d y=v_{+} d t$, the expression in Eq. (38) equals $(\hbar / m) T$ where $T$ is the time required for one round trip down and up. Thus, the depolarization rate is

$$
\begin{aligned}
\tau_{\text {dep }}^{-1} & =\frac{\left\langle j_{l}+j_{u}\right\rangle}{N}=\frac{m}{\hbar} \frac{\left\langle j_{l}+j_{u}\right\rangle}{T} \\
& =K^{2}\left(\frac{k_{x, \max }^{2}}{3}\right)\left(\frac{\sin ^{2} \theta_{l}}{k_{-l}^{4}}+\frac{\sin ^{2} \theta_{u}}{k_{-u}^{4}}\right) \frac{1}{T},
\end{aligned}
$$

where we have inserted Eq. (37) for the current in the last step. This shows that the loss current (37) of spin-flipped UCNs is the loss per round trip, i.e., for one bounce in the magnetic field. This interpretation is consistent with the interpretation in Ref. [14].

To compare to actual experiments storing polarized $(+)$ UCNs in a broad velocity range in three dimensions, we have to average Eq. (39) also over $v_{z}$ and $v_{y}$. Averaging over $v_{z}$ is trivial since Eq. (39) does not depend on $v_{z}$.

Averaging over $v_{y}$ can be achieved as follows. As a measure of $v_{y}$ for a stored UCN we could take its value at any height within the confinement range, but the most convenient choice of reference plane is the neutral plane at $y=y^{(\mathrm{n})}$ where the gravitational force is compensated by the magnetic force pushing upward, i.e., where $\left|\mu_{\mathrm{n}}\right| \frac{d B}{d y}=-m g$. This is the plane where the UCNs with the lowest energy for vertical motion reside. In our field model, a UCN with vertical velocity $v_{y}^{(n)}=0$ in the neutral plane floats or moves along the plane at constant speed. In actual confinement fields as in Ref. [14] they would follow closed or open paths on the curved neutral surface. For small values of $v_{+}^{(n)}$ the vertical motion is a classical harmonic oscillation with natural frequency $\omega_{0}=\left(\frac{d g_{+}}{d y}\right)^{1 / 2}$ where $g_{+}=g+\frac{\left|\mu_{\mathrm{n}}\right|}{m} \frac{d B}{d y}$ is the net downward acceleration. This implies that for small oscillations about the neutral plane the time for a round trip becomes $T=2 \pi / \omega_{0}=2 \pi\left(\frac{d g_{+}}{d y}\right)^{-1 / 2}$. For larger vertical velocities the oscillator potential is strongly anharmonic but the drop height $y_{0}$, used originally as a measure of energy for vertical motion, is unambiguously determined by $v_{+}^{(\mathrm{n})}$. Therefore, if we plot the depolarization rate (39) versus $v_{+}^{(\mathrm{n})}$, rather than $y_{0}$, the mean height of this curve in the range from $v_{+}^{(n)}=0$ to its maximum value for the stored UCN spectrum directly gives the average value of depolarization rate for a Maxwell spectrum. (The trap loading process used in an actual experiment may induce deviations from the Maxwell spectrum.)

Such a plot is presented in Fig. 3 where we have normalized $v_{+}^{(\mathrm{n})}$ to $v_{-0}^{(n)}$, the $y$ velocity for the spin-flipped state on the neutral plane for $v_{+}^{(\mathrm{n})}=0 . v_{-0}^{(\mathrm{n})}=2\left(\frac{\left|\mu_{\mathrm{n}}\right| B^{(\mathrm{n})}}{m}\right)^{1 / 2}$ is solely determined by the field magnitude $B^{(n)}$ on the neutral plane. The parameters used are: $y_{0, \max }=45 \mathrm{~cm}, B_{10}=0.005 \mathrm{~T}$ and 


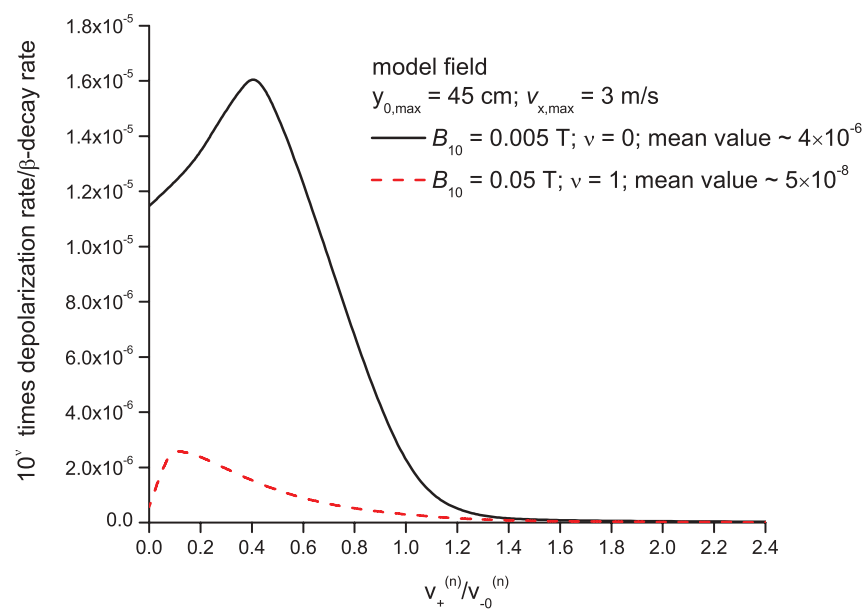

FIG. 3. (Color online) Ratio between mean depolarization rate, given by Eq. (39), and neutron $\beta$-decay rate (for a lifetime of $882 \mathrm{~s}$ ), plotted as a function of vertical velocity component $v_{+}^{(\mathrm{n})}$ in the neutral plane (where the gravitational and magnetic forces are balanced). $v_{+}^{(\mathrm{n})}$ is normalized with the constant $v_{-0}^{(\mathrm{n})}$ which is determined by the field magnitude in the neutral plane. The curve for $B_{10}=0.005 \mathrm{~T}$ is plotted to scale $(v=0)$ and the curve for $B_{10}=0.05 \mathrm{~T}$ is plotted with magnification factor $10^{1}(v=1)$. Their difference by about two orders of magnitude shows the strong suppression of depolarization by a stabilization field of sufficient strength. For a Maxwell spectrum, the mean height of the curves over the range of the abscissa, from 0 to 2.5 for $B_{10}=0.05 \mathrm{~T}$ and from 0 to 4.7 for $B_{10}=0.005 \mathrm{~T}$, directly determines the average over the full spectrum (here for $-3 \mathrm{~m} / \mathrm{s}<v_{x}<+3 \mathrm{~m} / \mathrm{s}$ and drop heights $y_{0}$ up to $450 \mathrm{~mm}$ ).

$0.05 \mathrm{~T}$, and $v_{x, \max }=3 \mathrm{~m} / \mathrm{s}$. For these parameters the mean depolarization rate, normalized to the $\beta$-decay rate $1 / \tau_{\mathrm{n}}$, is $\tau_{\mathrm{n}}\left\langle\tau_{\text {dep }}^{-1}\right\rangle=4 \times 10^{-6}$ for $B_{10}=0.005 \mathrm{~T}$ and about two orders of magnitude less for $B_{10}=0.05 \mathrm{~T}$.

It might come as a surprise that the largest contribution to the depolarization rate originates from UCNs with fairly low energy of vertical motion. They move through the field almost horizontally, with small vertical oscillations about the neutral plane. The result is plausible since these UCNs spend the largest fraction of time in the region where the field rotates rapidly in the reference frame of the laterally moving neutron.

In Sec. IV B we will analyze the same problem of depolarization in magnetic storage of UCNs using the semiclassical method and compare the two approaches. Next we apply the quantum approximation to analyze the depolarization in reflection of polarized neutrons on a nonmagnetic mirror immersed in a nonuniform magnetic field.

\section{Reflection on a nonmagnetic mirror in a magnetic field}

In Sec. I we mentioned two examples where the possible depolarization of UCNs in total reflection from a nonmagnetic wall, like copper, is of major importance: (a) UCN experiments on spin anisotropy parameters in neutron decay, such as the neutron-electron spin correlation coefficient $A$ [20,21]; and (b) UCN polarizers based on transmission through a high magnetic field. Depolarization in mirror reflection has been investigated theoretically in Ref. [17]. We will study aspects of this

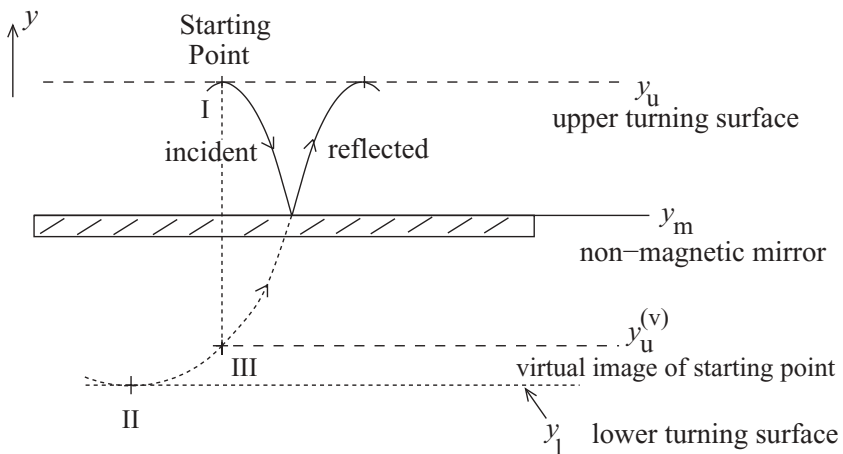

FIG. 4. Geometry of nonmagnetic mirror conceptually placed into the magnetic storage space at various levels $y_{m}$ between the upper and lower turning surfaces at $y_{u}$ and $y_{l}$ for a given UCN energy for vertical motion. The reflected spin-flipped wave consists of the two components in Eq. (42): (a) the particular solution $\beta_{p}$ which is the same as for a wave moving upward from the lower turning point at II in the absence of the mirror; (b) the homogeneous wave $\beta_{h}$ induced at the mirror surface by the wave incident from above, starting from the upper turning level at point I. The position III of the virtual image of I below the mirror determines a phase angle.

problem by imagining an ideal, non-magnetic mirror inserted horizontally into our magnetic model field at various heights $y_{m}$, as shown in Fig. 4, and comparing the depolarization per bounce on this mirror with that in the field without the mirror. The reflecting mirror surface at $y_{m}$ lies between the upper and lower turning points $y_{u}$ and $y_{l}$ for UCNs. We use index $m$ to denote the quantities at the reflecting mirror surface and have assumed that the UCNs have given lateral velocities $v_{x}$ and $v_{z}$ and a fixed energy for vertical motion.

An ideal UCN mirror has reflection amplitude $R=$ $\exp (-2 i \Sigma)$ and reflectivity $|R|^{2}=1$. The phase angle $\Sigma=$ $\cos ^{-1}\left(k_{+m} / k_{c}\right)$ depends on the limit $k_{c}$ of total reflection and, for the system at hand, on the vertical component of incident wave vector, $k_{+m}$, which is the same for both wave components, $\alpha$ and $\beta$. We will see that the depolarization at the mirror is independent of $\Sigma$, i.e., it should be virtually the same for any low loss (almost ideal) mirror material. On physical grounds, no abrupt change of depolarization probability $|\beta|^{2}$ is expected since the dwell time of $\sim 10^{-8} \mathrm{~s}$ inside the wall is much shorter than the Larmor precession period ( $1 \mu \mathrm{s}$ for $B=34 \mathrm{mT}$ ) which sets the time scale for any change.

Figure 4 shows the geometry. As in the previous sections, we assume that the particle started out from an upper turning level $y_{u}$ in a pure $(+)$ spin state. On incidence at $y=y_{m}$ its wave function has acquired a depolarized component given by Eq. (32):

$$
\beta\left(y_{m}\right)=k_{-}^{-1 / 2}\left(y_{m}\right) P\left(y_{m}\right) \exp \left(-i \Phi_{+}\left(y_{m}\right)\right),
$$

where $P$ has been defined in Eq. (30). The evolution of $\beta(y)$ following reflection is determined by the inhomogeneous differential equation (23):

$$
\beta^{\prime \prime}(y)+k_{-}^{2}(y) \beta(y)=-\left[i k_{+} \theta^{\prime}(y)-K k_{x} \sin \theta(y)\right] \alpha(y),
$$

where we replaced the \pm sign in Eq. (23) by + since the reflected wave is moving upward. 
The solution to Eq. (41) can be written as a superposition

$$
\beta(y)=\beta_{p}(y)+\beta_{h}(y)
$$

of a particular solution, $\beta_{p}(y)$, and the general solution $\beta_{h}(y)$ of the homogeneous equation

$$
\beta_{h}^{\prime \prime}(y)+k_{-}^{2}(y) \beta_{h}(y)=0 .
$$

The amplitude multiplying $\beta_{h}(y)$ is adjusted to satisfy initial conditions.

A particular solution $\beta_{p}$ for downward motion has been given as Eq. (32). For the mirror-reflected beam we need the corresponding solution for upward motion. It follows from the discussion in Sec. III B1 that this particular solution is

$$
\beta_{p}(y)=\xi k_{-}^{-1 / 2}(y) P^{*}(y) \exp \left(i \Phi_{+}(y)\right) .
$$

The phase factor $\xi=\exp \left(-i \Phi_{t}\right)$, with $\Phi_{t}=\int_{y_{u}}^{y_{l}}\left(k_{+}-k_{-}\right) d y$, arises as follows: For the mirror reflection problem we choose a time axis with $t=0$ at the upper turning point $y_{u}$ and increasing as the motion proceeds. However, the corresponding time $t^{\prime}$, chosen in Sec. III B1 for the upward motion, starts from $t^{\prime}=0$ at $y_{l}$ (not from $y_{u}$ ). The times required to reach a given particle position are related through $t^{\prime}=-t+\frac{T}{2}$, where, as before, $T$ is the time for a round trip down and up. The phase factor $\xi$ arises due to this difference in the time coordinates.

The homogeneous equation (43) has two solutions but only one, $\beta_{h}(y) \sim \exp \left(+i k_{-m}\left(y-y_{m}\right)\right)$, corresponds to upward wave propagation, as required for the reflected wave. The plane wave form is valid only near the mirror surface where $k_{-}(y)$ is considered constant on the scale of the neutron wavelength. Thus, in the framework of the WKB approximation, we use the homogeneous solution

$$
\beta_{h}(y)=C k_{-}^{-1 / 2}(y) \exp \left(+i \Phi_{-}(y)\right),
$$

where the constant $C$ is to be adjusted to match the outgoing wave (42) to the wave

$$
\beta_{m}=r k_{-}^{-1 / 2}\left(y_{m}\right) P\left(y_{m}\right) \exp \left(-i \Phi_{+}\left(y_{m}\right)\right)
$$

excited by the incoming beam. Expression (46) is the incoming wave (32) multiplied by the phase factor $r=R \exp \left(-2 i \Phi_{-m}\right)$ where $R$ is the reflection amplitude. The factor $\exp \left(-2 i \Phi_{-m}\right)$ is due to a shift of time scales similar to that defined following Eq. (44) but now referring to mirror reflection with start from the upper turning point $y_{u}$ versus start from its virtual image at $y_{u}^{(v)}$ below the mirror surface, as shown in Fig. 4.

Now we match the outgoing wave $\beta_{p}(y)+\beta_{h}(y)$ [from Eqs. (44) and (45)] to the wave $\beta_{m}$ at the mirror surface $y_{m}$ [from Eq. (46)] to determine the constant $C$, with the result

$$
\begin{aligned}
C & =\left(\beta_{m}-\beta_{p m}\right) \exp \left(-i \Phi_{-m}\right) \\
& =\left[r P_{m} \exp \left(-i \Phi_{+m}\right)-\xi P_{m}^{*} \exp \left(+i \Phi_{+m}\right)\right] \exp \left(-i \Phi_{-m}\right) .
\end{aligned}
$$

Thus the mirror reflected wave becomes

$$
\begin{aligned}
\beta(y)= & \beta_{p}(y)+\beta_{h}(y) \\
= & k_{-}^{-1 / 2}(y)\left\{\xi P^{*}(y) \exp \left(i \Phi_{+}(y)\right)\right. \\
& +\left[r P_{m} \exp \left(-i \Phi_{+m}\right)-\xi P_{m}^{*}\right. \\
& \left.\left.\times \exp \left(+i \Phi_{+m}\right)\right] \exp \left(-i \Phi_{-m}\right) \exp \left(i \Phi_{-}(y)\right)\right\} .
\end{aligned}
$$

The first term in the braces represents an outgoing wave with wave number $k_{+}$and the second one with wave number $k_{-}$. To calculate the outgoing current we also need the derivative

$$
\begin{aligned}
\frac{d \beta}{d y}= & k_{-}^{-1 / 2}(y)\left\{i k_{+} \xi P^{*}(y) \exp \left(i \Phi_{+}(y)\right)\right. \\
& +i k_{-}\left[r P_{m} \exp \left(-i \Phi_{+m}\right)-\xi P_{m}^{*} \exp \left(+i \Phi_{+m}\right)\right] \\
& \left.\times \exp \left(-i \Phi_{-m}\right) \exp \left(i \Phi_{-}(y)\right)\right\} .
\end{aligned}
$$

The current propagating in the upward direction is obtained from [22]

$$
\frac{m}{\hbar} j_{+}(y)=-\operatorname{Re}\left[i \beta^{*}(y)\left(\frac{d \beta}{d y}\right)\right],
$$

and the result is a sum of slowly varying terms representing the measurable depolarization. There are also fast oscillating terms with phase $\pm \Phi(y)= \pm\left[\Phi_{+}(y)-\Phi_{-}(y)\right]$ which would be averaged to zero by a detector of spin-flipped UCNs except within a narrow range of order $2 \pi /\left(k_{-m}-k_{+m}\right)$, i.e., of a few wavelengths above the mirror. With the same proviso, mixed terms $\sim \xi^{*}$ (or $\xi^{*} r$ ) can also be dropped since the phase factors $\xi$ and $r$ depend sensitively on the exact position of the mirror and the exact distance between upper and lower turning points. In practice these quantities are blurred by geometrical imperfections as well as the finite spread in UCN energy. Thus $\xi$ and $r$ can be considered statistically independent of one another. As a result we obtain for the measurable average current

$$
\begin{aligned}
\left\langle\frac{m}{\hbar} j_{+}(y)\right\rangle= & \frac{k_{+}}{k_{-}}|P(y)|^{2}+2\left|P\left(y_{m}\right)\right|^{2} \\
= & \frac{\left(k_{+} \theta^{\prime}\right)^{2}+\left(K k_{x} \sin \theta\right)^{2}}{\left(k_{-}^{2}-k_{+}^{2}\right)^{2}} \\
& +2\left(\frac{k_{-m}}{k_{+m}}\right) \frac{\left(k_{+m} \theta_{m}^{\prime}\right)^{2}+\left(K k_{x} \sin \theta_{m}\right)^{2}}{\left(k_{-m}^{2}-k_{+m}^{2}\right)^{2}} .
\end{aligned}
$$

To measure the depolarization per one complete bounce on the mirror from upper turning point down and back up to the upper turning point, we insert $y=y_{u}$ for the detector position and obtain

$$
\begin{aligned}
\left\langle\frac{m}{\hbar} j_{+}\left(y_{u}\right)\right\rangle= & \left(\frac{K k_{x}}{k_{-u}^{2}} \sin \theta_{u}\right)^{2} \\
& +2\left(\frac{k_{-m}}{k_{+m}}\right) \frac{\left(k_{+m} \theta_{m}^{\prime}\right)^{2}+\left(K k_{x} \sin \theta_{m}\right)^{2}}{\left(k_{-m}^{2}-k_{+m}^{2}\right)^{2}} .
\end{aligned}
$$

This corresponds to expression (36) for one bounce in the magnetic field. In Eq. (52) we have used $k_{+u}=k_{+}\left(y_{u}\right)=0$ and we note that in typical cases the first term in Eq. (52) is negligible.

We have assumed that the UCNs are incident on the mirror from above, i.e., are confined to the space $y_{m}<y<y_{u}$. If, instead, they impinge from below at $y=y_{m}$ (now the lower mirror surface) and are confined to the space $y_{l}<y<y_{m}$, the expressions (51) and (52) (now for $j_{-}$, not $j_{+}$) remain the same except that in Eq. (52) the index $u$ is replaced by $l$.

We will discuss these results in greater detail in Secs. IV C and VI. 


\section{SEMICLASSICAL APPROACH}

\section{A. Basic equations}

The semiclassical Schrödinger equation describes the particle in time $t$, rather than in space coordinates. The particle is assumed to follow a known classical path, so the field variable $\boldsymbol{B}$ is considered a known function of $t$. We again choose the quantization axis along the position-dependent direction of $\boldsymbol{B}$, for which the mutually orthogonal basis vectors $\chi^{+}$and $\chi^{-}$ are given in Eq. (6) and the wave function for the $(+)$ and $(-)$ state is

$$
\chi(t)=\alpha(t) \chi^{+}(t)+\beta(t) \chi^{-}(t) .
$$

But these quantities are now considered to be functions of $t$, rather than of space variables. As before, the spin flip probability $|\beta(t)|^{2}$ is considered small compared to the probability $|\alpha(t)|^{2} \approx 1$ to find the neutron in the storable $(+)$ spin state.

In this approximation the Schrödinger equation reads [14]

$$
\begin{aligned}
i \hbar \frac{d}{d t}\left(\alpha \chi^{+}+\beta \chi^{-}\right) & =\mathcal{H}_{m}\left(\alpha \chi^{+}+\beta \chi^{-}\right) \\
& =\left|\mu_{\mathrm{n}}\right| B\left(\alpha \chi^{+}-\beta \chi^{-}\right),
\end{aligned}
$$

where the Hamiltonian $\mathcal{H}_{m}$ for spin interaction was given in Eq. (3). We denote time derivatives by a dot and, using the notation and relations of Sec. III A and from $\phi=-K x, \dot{\phi}=$ $v_{x} \frac{d \phi}{d x}=-v_{x} K$, obtain

$$
\begin{aligned}
\dot{\chi}^{+} & =\left(\begin{array}{c}
\left(i s K v_{x}+\frac{1}{2} c \dot{\theta}\right) e_{-} \\
\frac{1}{2} s \dot{\theta}
\end{array}\right) \\
& =\frac{1}{2}\left(\dot{\theta}+i K v_{x} \sin \theta\right) e_{-} \chi^{-}+i K s^{2} v_{x} \chi^{+}
\end{aligned}
$$

and

$$
\begin{aligned}
\dot{\chi}^{-} & =\left(\begin{array}{c}
-\frac{1}{2} s \dot{\theta} \\
\left(\frac{1}{2} c \dot{\theta}-i s K v_{x}\right) e_{+}
\end{array}\right) \\
& =-\frac{1}{2}\left(\dot{\theta}-i K v_{x} \sin \theta\right) e_{+} \chi^{+}-i K s^{2} v_{x} \chi^{-} .
\end{aligned}
$$

Inserting into Eq. (54) and keeping only the dominant contributions we get for the terms with $\chi^{+}$

$$
\dot{\alpha}+\frac{i \omega_{L}}{2} \alpha=0
$$

and for those with $\chi^{-}$

$$
\dot{\beta}-\frac{i \omega_{L}}{2} \beta=-\frac{\alpha}{2}\left(\dot{\theta}+i K v_{x} \sin \theta\right) e_{-},
$$

where $\omega_{L}=2\left|\mu_{\mathrm{n}}\right| B / \hbar$ is the Larmor frequency. In practice, $\omega_{L} \gg K v_{x}$. Equations (57) and (58) correspond to Eqs. (14) and (15) of Ref. [14], but Eq. (58) contains the new phase factor $e_{-}=\exp (-i \phi)=\exp (i K x)$ [as the quantum equivalent, Eq. (12), does] and the term dependent on $v_{x}$.

\section{B. Depolarization in the magnetic field}

Equation (57) is solved by

$$
\alpha(t)=\exp \left(-\frac{i \Theta}{2}\right)
$$

where $\Theta=\int_{t_{s}}^{t} \omega_{L}\left(t^{\prime}\right) d t^{\prime}$ is twice the phase angle accumulated since the start time $t_{s}$. As for the quantum case, we assume that the motion starts at the upper or lower turning point with velocity $\boldsymbol{v}_{s}=\left(v_{x}, v_{+s}, v_{z}\right)=\left(v_{x}, 0, v_{z}\right)$, where $v_{x}=$ const. and the constant $z$ component $v_{z}$ does not induce depolarization for our field model. $v_{+s}=v_{+}\left(t_{s}\right)=0$ implies $\dot{\theta}=0$ at the start.

As in Ref. [14], Eq. (58) is solved using the ansatz $\beta(t)=G(t) \exp (i \Theta / 2)$, where $G(t)$, the new measure of depolarization amplitude [14], satisfies the relation

$$
\dot{G}=-\frac{1}{2}\left(\dot{\theta}+i K v_{x} \sin \theta\right) \exp (-i \Theta) \exp (i K x)
$$

Since $x=v_{x} t$ the factor $\exp (i K x)=\exp \left(i K v_{x} t\right)$ is time dependent and therefore $i \hbar \frac{d}{d t} \exp \left(i K v_{x} t\right)$ contributes to the energy. However, as noted before, $K v_{x}$ is much smaller than $\omega_{L}$ and can be neglected in the same way small terms are neglected in the WKB approximation.

Equation (60) is readily integrated by parts:

$$
\begin{aligned}
G(t) & =\int_{t_{s}}^{t} \dot{G}\left(t^{\prime}\right) d t^{\prime} \\
& =-\frac{i}{2 \omega_{L}}\left(\dot{\theta}+i K v_{x} \sin \theta\right) \exp (-i \Theta) \exp (i K x)
\end{aligned}
$$

As for the quantum analog (28) of Eq. (61), the right-hand side of Eq. (61) does not include a term for the lower integration limit $t_{s}$. As we will see, this leads to results matching those of the quantum approach. We again can argue, as in the discussion of Eq. (28), that in view of the Airy-function character of the actual wave solution around the turning region, the initial time $t_{s}$ is a blurred quantity and the contribution from the lower limit of integration averages to zero. But a rigorous justification may be impossible within this semiclassical mix of ingredients as incongruent as classical and quantum mechanics are.

We identify $|G(t)|^{2}$ with the probability $p$ of finding the neutron in the spin-flipped state [14]

$$
p=\frac{\dot{\theta}^{2}+K^{2} v_{x}^{2} \sin ^{2} \theta}{4 \omega_{L}^{2}}=\frac{k_{+}^{2} \theta^{\prime 2}+K^{2} k_{x}^{2} \sin ^{2} \theta}{\left(k_{-}^{2}-k_{+}^{2}\right)^{2}} .
$$

In the last step of Eq. (62) we have used $\omega_{L}=\frac{\hbar}{2 m}\left(k_{-}^{2}-k_{+}^{2}\right)$, and $\dot{\theta}^{2}=v_{+}^{2}\left(\frac{d \theta}{d y}\right)^{2}=\left(\frac{\hbar}{m}\right)^{2} k_{+}^{2} \theta^{\prime 2}$, since in our field model $\theta$ depends only on $y$.

The result (62) agrees with Eq. (34) for the function $(m / \hbar) j_{-}$which had also been identified as the depolarization probability. Therefore we have full agreement also for the mean depolarization per bounce (37) and for the depolarization rate given in Eq. (39).

In the next section we will see that the semiclassical and quantum approaches do not always produce exactly the same results, although a strong correlation between the two will be found also in this case. 


\section{Reflection from a nonmagnetic mirror in a magnetic field}

The semiclassical analysis of the mirror problem follows the same path as for the quantum case analyzed in Sec. III C. The crucial step again is matching the superposition of particular plus homogeneous solution for the ascending reflected beam,

$$
\beta(t)=\beta_{p}(t)+\beta_{h}(t),
$$

to the wave $\beta_{m}$ induced by the incident beam at the mirror surface at $t=t_{m}$.

In Eq. (63), $\beta_{h}=C \exp \left(\frac{-i \Theta}{2}\right)$ is the general solution of the homogeneous equation $\dot{\beta}_{h}+\frac{i \omega_{L}}{2} \beta_{h}=0$, which corresponds to the inhomogeneous equation (58) with the direction of time reversed as described following Eq. (44). Using the same sequence of terms as in Eq. (63) and the notation of Sec. III C, the matching condition $\beta\left(t_{m}\right)=\beta_{p}\left(t_{m}\right)+\beta_{h}\left(t_{m}\right)$ reads

$$
\begin{aligned}
& \frac{i \zeta_{1}}{2 \omega_{L m}}\left(\dot{\theta}_{m}-i K v_{x} \sin \theta_{m}\right) \exp \left(+\frac{i \Theta_{m}}{2}\right) \\
& =\frac{i \zeta_{2}}{2 \omega_{L m}}\left(\dot{\theta}_{m}-i K v_{x} \sin \theta_{m}\right) \exp \left(+\frac{i \Theta_{m}}{2}\right) \\
& \quad+C \exp \left(-\frac{i \Theta_{m}}{2}\right),
\end{aligned}
$$

where we have divided out the common factor $\exp \left(-i K v_{x} t_{m}\right)$. $\omega_{L m}$ is the Larmor frequency at the mirror position and $\zeta_{1}, \zeta_{2}$ are statistically independent unitary phase factors similar to their quantum analogs $\xi, r$ in Eqs. (44) and (46). Solving for the constant $C$ of the homogeneous term and inserting into Eq. (63) gives

$$
\begin{aligned}
\beta(t)= & \exp \left(-i K v_{x} t\right)\left[\frac{i}{2 \omega_{L}}\left(\dot{\theta}-i K v_{x} \sin \theta\right) \exp \left(+\frac{i \Theta}{2}\right)\right. \\
& +\left(\zeta_{1}-\zeta_{2}\right)\left(\frac{i}{2 \omega_{L m}}\right)\left(\dot{\theta}_{m}-i K v_{x} \sin \theta_{m}\right) \\
& \left.\times \exp \left(+i \Theta_{m}\right) \exp \left(-\frac{i \Theta}{2}\right)\right]
\end{aligned}
$$

Finally, taking the square magnitude of Eq. (65) and performing the same statistical averaging as for Eq. (50), which includes setting $\left\langle\left|\zeta_{1}\right|^{2}\right\rangle=\left\langle\left|\zeta_{2}\right|^{2}\right\rangle=1$ and $\left\langle\zeta_{1} \zeta_{2}^{*}\right\rangle=0$, we find for the probability of depolarization

$$
\begin{aligned}
p(t)=\left\langle|\beta(t)|^{2}\right\rangle= & \frac{1}{4 \omega_{L}^{2}}\left[\dot{\theta}^{2}+\left(K v_{x} \sin \theta\right)^{2}\right] \\
& +\frac{1}{2 \omega_{L m}^{2}}\left[\dot{\theta}_{m}^{2}+\left(K v_{x} \sin \theta_{m}\right)^{2}\right] .
\end{aligned}
$$

To facilitate comparison with the quantum result (51) we convert from time to space dependent variables $\left(\dot{\theta} \rightarrow \theta^{\prime}=\right.$ $\left.\frac{d \theta}{d y}\right)$, as for Eq. (62), and obtain

$$
\begin{aligned}
p(t)= & \frac{\left(k_{+} \theta^{\prime}\right)^{2}+\left(K k_{x} \sin \theta\right)^{2}}{\left(k_{-}^{2}-k_{+}^{2}\right)^{2}} \\
& +\frac{2\left[\left(k_{+m} \theta_{m}^{\prime}\right)^{2}+\left(K k_{x} \sin \theta_{m}\right)^{2}\right]}{\left(k_{-m}^{2}-k_{+m}^{2}\right)^{2}} .
\end{aligned}
$$

This is the same expression as Eq. (51) except that the factor $k_{-m} / k_{+m}$ for the second term on the right-hand side is missing.

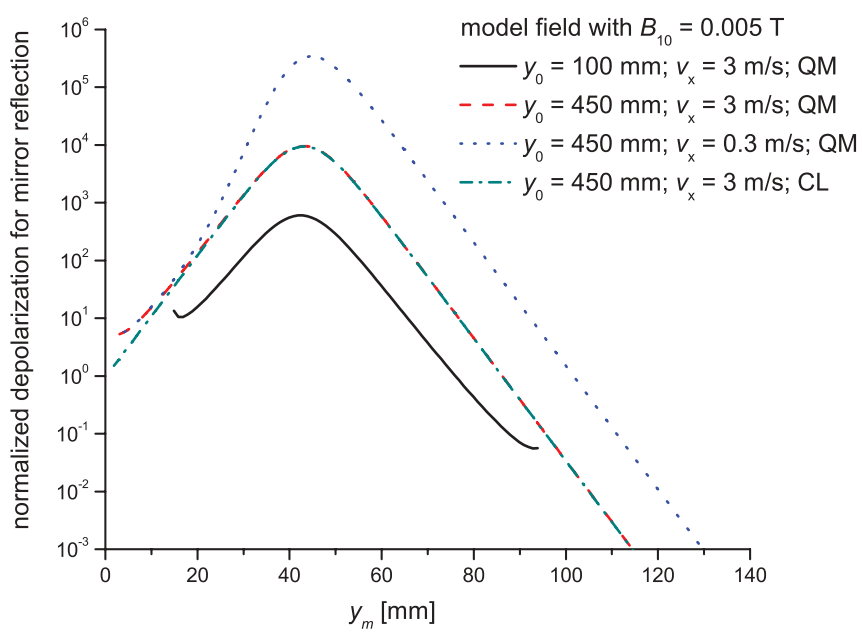

FIG. 5. (Color online) Normalized depolarization as a function of mirror position $y_{m}$. We use Eq. (52) for the quantum treatment (QM) and Eq. (68) for the semiclassical approach. The plotted values are normalized by dividing Eq. (68) by Eq. (36), the depolarization due to the field alone, and by the ratio of duration of one bounce with and without the mirror. This figure shows a strong enhancement of depolarization due to the mirror. The enhancement factor depends on incident UCN energy (which increases with larger fall height $y_{0}$ ) and on the gradient $\theta_{m}^{\prime}$ of field angle $\theta$ at the mirror position. $\theta_{m}^{\prime}$ is largest in the region where the curves have their peak value which is of order $10^{4}$ for $v_{x}=3 \mathrm{~m} / \mathrm{s}$. For the examples shown, the turning point levels are: $y_{u}=96.86 \mathrm{~mm}, y_{l}=14.31 \mathrm{~mm}$ for $y_{0}=100 \mathrm{~mm}$, and $y_{u}=445.82 \mathrm{~mm}, y_{l}=0.58 \mathrm{~mm}$ for $y_{0}=450 \mathrm{~mm}$.

Except for very low energy UCNs hovering in the magnetic field, and for mirror position at a turning point, this factor is close to 1 .

For a complete bounce on the mirror, starting from, and ending at, the upper turning point level $y_{u}$ we obtain

$$
p=\frac{\left(K k_{x} \sin \theta_{u}\right)^{2}}{k_{-u}^{4}}+\frac{2\left[\left(k_{+m} \theta_{m}^{\prime}\right)^{2}+\left(K k_{x} \sin \theta_{m}\right)^{2}\right]}{\left(k_{-m}^{2}-k_{+m}^{2}\right)^{2}} .
$$

Figure 5 shows the depolarization per bounce on the mirror in our model field as a function of mirror position $y_{m}$. For the quantum treatment (QM) this probability is given by Eq. (52) and for the semiclassical approach (CL) by Eq. (68). To separate the role of the nonmagnetic mirror in the field from the depolarization due to the field alone we have divided Eq. (68) by Eq. (36), the depolarization due to the field alone, and by the ratio of duration of one bounce with and without the mirror. Figure 5 shows a strong enhancement due to the mirror. The enhancement factor depends on incident UCN energy (which increases with larger fall height $y_{0}$ ) and on the gradient $\theta_{m}^{\prime}$ of field angle at the mirror position. $\theta_{m}^{\prime}$ is largest in the region where the curves have their peak. The enhancement reaches four orders of magnitude for $y_{0}=0.45 \mathrm{~m}$ and $v_{x}=3 \mathrm{~m} / \mathrm{s}$, and six decades for $v_{x}=0.3 \mathrm{~m} / \mathrm{s}$. In the latter comparison, the role of lateral velocity component $v_{x}$ appears so large because the depolarization in the field alone vanishes for $v_{x}=0$ in our approximation (36) (and is negligibly small in higher-order approximations [14]), while the mirror depolarization remains finite for $v_{x}=0$. 
The quantum and semiclassical results, compared for identical parameters by the dashed and dash-dotted curves in Fig. 5, are quite similar. For the given parameters they differ by a few percent at most if we exclude the range within $\sim 5 \mathrm{~mm}$ from a turning point.

As for the quantum approach, the results (67) and (68) remain the same [except for the index $u$ in Eq. (68) changing to $l$ ] if incidence on the mirror from above is replaced by incidence from below, with start from the lower turning point $y_{l}$ rather than from $y_{u}$.

In the next section we will show that for low UCN energies these analytical results can also be obtained by numerical integration of the basic differential equation. Numerical integration does not rely on the WKB approximation. However, the lengthy integration over many oscillations of the wave function is plagued with the rounding errors and the error due to the finite step size. Reasonable agreement of the two methods would be an indication that the results are reliable.

\section{NUMERICAL INTEGRATION}

\section{A. Magnetic confinement}

The equation of motion of the wave function $\beta(y)$ for spin flip is given by the second-order inhomogeneous differential equation (23),

$$
\beta^{\prime \prime}(y)+k_{-}^{2}(y) \beta(y)=-\left[ \pm i k_{+} \theta^{\prime}(y)-K k_{x} \sin \theta(y)\right] \alpha(y),
$$

with Eq. (16)

$$
\alpha(y)=k_{+}^{-1 / 2}(y) \exp \left( \pm i \Phi_{+}(y)\right)
$$

and the $+(-)$ sign refers to motion upward (downward). Using the fourth-order Runge Kutta process, Eq. (23) may be integrated numerically, starting from an initial point $y_{i}$ slightly above (below) the turning point $y_{s}$. Key is the suitable choice of initial values $\beta\left(y_{i}\right)$ and $\beta^{\prime}\left(y_{i}\right)$.

We choose the WKB solution which is given by Eq. (32) for downward motion. Its extension to include also the upward path reads

$$
\beta(y)=k_{-}^{-1 / 2}(y) P_{ \pm}(y) \exp \left( \pm i \Phi_{+}(y)\right)
$$

with derivative

$$
\beta^{\prime}(y)= \pm i k_{+}(y) k_{-}^{-1 / 2}(y) P_{ \pm}(y) \exp \left( \pm i \Phi_{+}(y)\right),
$$

where $P_{-}(y)=P(y)$ and $P_{+}(y)=P^{*}(y) . P(y)$ has been defined in Eq. (30). As discussed following Eq. (19), near a turning point $y_{s}$ Eq. (69) is based on the asymptotic form of the Airy function wave solution $\alpha(y)=C_{1} \operatorname{Ai}\left(-a_{s}\left|y-y_{s}\right|\right)$, where the coefficient $C_{1}=2\left( \pm i \pi / a_{s}\right)^{1 / 2}$ is adjusted to match Eq. (16) asymptotically. The constants $g_{+s}$ and $a_{s}$ were defined following Eq. (19). Since $g_{+}$varies slowly, the constant value $g_{+s}$ is a good approximation over hundreds of oscillations of the Airy function, starting from $y=y_{s}$.

Using initial values Eqs. (69) and (70), Eq. (23) may be integrated numerically up to a point slightly before the next turning point, $y_{s, \text { next }}$, is reached. The method fails at $y_{s, \text { next }}$ itself due to the divergence of $k_{+}^{-1 / 2}$. We calculate the

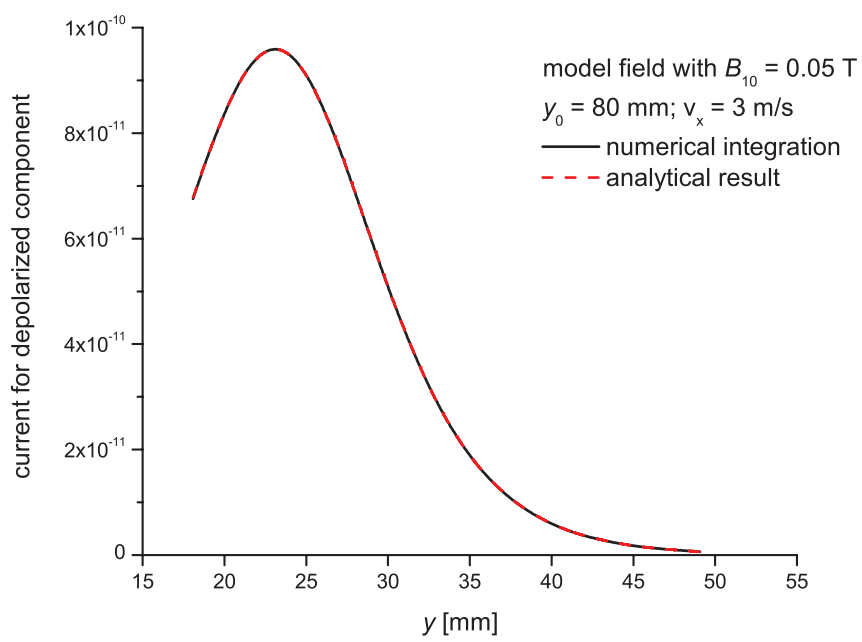

FIG. 6. (Color online) Depolarization current for downward motion in the magnetic field for parameters $y_{0}=8 \mathrm{~cm}, B_{10}=0.05 \mathrm{~T}$, and $v_{x}=3 \mathrm{~m} / \mathrm{s}$. Direct numerical integration, represented by the solid curve, coincides with the analytic result shown by the dashed curve, with a maximum deviation of $1 \%$ over the entire range from upper to lower turning point ( $y_{u}=49.56 \mathrm{~mm}, y_{l}=18.08 \mathrm{~mm}$ ).

current $(m / \hbar) j(y)= \pm \operatorname{Re}\left[\beta^{*}(y) \beta^{\prime}(y) / i\right]$ at every point along the integration path and Fig. 6 shows the result of downward integration for parameters $y_{0}=8 \mathrm{~cm}, B_{10}=0.05 \mathrm{~T}, v_{x}=$ $3 \mathrm{~m} / \mathrm{s}$ and starting point at $\left|y_{i}-y_{s}\right|=10 / a_{s}$. We have tested that the solution is stable in a wide range of initial position from $a_{s}\left|y_{i}-y_{s}\right| \sim 2$ to 20 .

Figure 6 shows that the numerical result coincides with the analytical solution (69), with maximum deviations of $\sim 1 \%$ over the entire range including the far endpoint $y_{l}$. This consistency at the lower turning point is vital since the current leaving there from the storage space is identified with the depolarization probability for the move from top to bottom. To be specific, this depolarization probability $(m / \hbar) j_{-}\left(y_{l}\right)$ is obtained by extrapolating the numerical solution over the short distance of order $a_{s, \text { next }}^{-1}$ to the next endpoint $y_{s, \text { next }}$ with the result $(m / \hbar) j_{-}\left(y_{l}\right)=6.80 \times 10^{-11}$ for the case shown in Fig. 6. The extrapolation at $y_{l}=18.08 \mathrm{~mm}$ is straightforward since the current shows a smooth behavior in the entire integration range. The same level of agreement within $\sim 1 \%$ is obtained for the upward motion which is represented by the same curve of Fig. 6, but the extrapolation for the loss current is now made at the upper end $y_{u}=$ $49.08 \mathrm{~mm}$ (and this contribution is much smaller in the case shown, but it is comparable for smaller drop heights). We found agreement within $1 \%$ between the various methods also for other parameters $y_{0}, B_{10}$, and $v_{x}$ within the range of interest.

There is one exception. For the strictly vertical mode of motion analyzed in Ref. [14], where $v_{x}=0$, the analytical solution vanishes and the numerical solution for the intercept is at least four orders of magnitude smaller than for $v_{x}=3 \mathrm{~m} / \mathrm{s}$. In this case the numerical precision is insufficient to determine a reliable value of depolarization probability. However, this is inconsequential since extremely small values of $v_{x}$ make a 
negligible contribution to the mean depolarization rate in a broad UCN spectrum.

We add one remark. If the explicit analytical solutions (69) and (70) for $\beta$ and $\beta^{\prime}$ were not known, we could use, for the numerical integration, initial values derived solely from the properties of the Airy function solution near the turning points. In these regions the second derivative $\beta^{\prime \prime}$ in Eq. (23) is negligible compared with $k_{-}^{2} \beta$ since $k_{-}^{2} \gg a_{s}^{3}\left|y-y_{s}\right|=k_{+}^{2}$. Therefore, $\beta(y) \approx-\left[ \pm i k_{+} \theta^{\prime}(y)-K k_{x} \sin \theta(y)\right] \alpha(y) / k_{-}^{2}(y)$ is a good approximation. It differs from Eq. (69) only by the multiplier $\left[1-k_{+}^{2}(y) / k_{-}^{2}(y)\right]$ which is close to 1 at either turning point since $k_{+}$vanishes there. It turns out that these modified initial values give the same results for $\beta(y)$ at both endpoints, and therefore the same depolarization probability, as the more exact method. However, the function $\beta(y)$ will be somewhat different in the range between the endpoints, where $\beta^{\prime \prime}$ cannot be neglected.

We may summarize the results on depolarization in magnetic storage as follows: The fact that the three methods used agree (quantum and semiclassical analysis as well as direct numerical integration) appears to be a good indication that the approximations made were justified.

\section{B. Mirror reflection}

For UCN reflection from a nonmagnetic mirror in a magnetic field the analytical results given in Eqs. (52) and (68) are similar but not identical. We used numerical integration for the reflected beam, starting from initial conditions (46) at the mirror surface and note that in this case the extrapolation to the next turning point is less straightforward since the curve for current shows, superimposed on a smooth variation, fast oscillations due to beating between two wave components: one propagating with wave number $k_{+}$and the other with $k_{-}$. This is expected for the superposition of these two waves in Eq. (48). The numerical results are generally consistent with the analytical expressions but not precise enough to decide whether the semiclassical approximation (68) or the quantum approximation (52) is more reliable.

\section{DISCUSSION}

Depolarization in high precision neutron lifetime experiments using magnetic confinement must either be negligible or else very small and quantitatively understood. Using two analytical methods based on Ref. [14] and direct numerical integration we have analyzed the depolarization per bounce and the depolarization rate (per s) for UCNs stored in a model magnetic field configuration. Our magnetic field model is similar to the system envisaged for the "bathtub" project [14] which uses a Halbach array of permanent magnets. Our model is simplified to a configuration with translational symmetry in both horizontal directions ( $z$ and $x$ ) and an ideal Halbach field whose magnitude depends only on the vertical distance $y$ from the horizontal magnet surface. The parameters for the Halbach field are the same as those proposed in Ref. [14]. Our analysis shows that depolarization is mainly caused by the rotating Halbach field the UCNs see as they move through the field with finite lateral velocity component $v_{x}$, not by the small field ripple due to imperfections of the Halbach system. Therefore we do not expect the simplification of the model to affect the depolarization in a significant way.

However, the role of the additional horizontal stabilization field $\boldsymbol{B}_{1}$ perpendicular to the Halbach field $\boldsymbol{B}_{H}$ is critical. It has to be strong enough to suppress depolarization to an acceptable level. The main purpose of this work was to determine tolerance limits for its magnitude $B_{1}$.

Our analysis extends that of Ref. [14] by including arbitrary UCN orbits in 3D space whereas the analysis in Ref. [14] was restricted to purely vertical motion. As a main result of the extension we find that the lateral $x$ component of motion in the plane of the Halbach field makes the dominant contribution to depolarization while the depolarization due to the vertical motion is insignificant. As a result, some previous estimates of depolarization probability may have been overoptimistic. For the parameters of Ref. [14] (0.05$0.1 \mathrm{~T}$ for $B_{10}$ ) we estimate on the basis of Fig. 3 that even a measurement of the neutron lifetime with precision $10^{-5}$ should be possible (disregarding other potential limitations) but the safety margin may be smaller than previously expected.

Systems with smaller stabilization field, as possibly that of Ref. [11], where $B_{1}$ has not been specified, may require a separate analysis since they use a cylindrical rather than planar field distribution. However, the main result of the present work is independent of geometrical details: The depolarization loss is determined, not by the largest rotation frequency of $\boldsymbol{B}$ as seen by the UCNs as they traverse the field, but by the conditions at the turning points where the spin-flipped neutrons can leave the storage system.

This is an important point which may appear to contradict the common view, but is also implied by the work of Ref. [14]. Our interpretation of the present results is as follows: The measurable depolarization is not directly caused by critical spots within the storage volume, where the $\boldsymbol{B}$ field has a small magnitude $B$ and rotates fast in the moving reference frame. All that matters for depolarization are the field conditions at the turning points where the storable UCNs are reflected back into the storage space while the spin-flipped fraction leaves the trap. The loss current in Eq. (37) is determined by the value of $K^{2} k_{x}^{2} \sin ^{2} \theta / k_{-}^{4}$ at such a surface. This factor is large for fast field variation $\left(\omega \sim v_{x} / L \sim K k_{x}\right)$ seen by the neutron moving in a horizontal direction (parallel to the turning surface), as well as for small $B$ since $k_{-}^{2}$ from Eq. (31) is directly proportional to $B$ at a turning point where $k_{+}=0$. It also increases quadratically with $\sin \theta=B_{H} / B$, i.e., with the magnitude $B_{H}$ of the Halbach field at a turning surface. If a broad spectrum of UCNs is stored the critical points will be of importance in the sense that some, usually UCNs with very low energy of vertical motion, may have a turning surface near such areas and therefore make a large contribution. In fact, the lowenergy UCNs make the largest contribution to depolarization seen in the peaks in Fig. 3, and they are the reason why a larger stabilization field $B_{1}$ suppresses the net depolarization very effectively. 
Figure 3 shows $\tau_{\mathrm{dep}}^{-1} / \tau_{\mathrm{n}}^{-1}$, the depolarization rate divided by the $\beta$-decay rate, as a function of normalized vertical velocity $v_{+}^{(\mathrm{n})}$ in the neutral plane, where gravity and the magnetic force are balanced. Both curves, for $B_{10}=0.05 \mathrm{~T}$ and for $B_{10}=$ $0.005 \mathrm{~T}$, are peaked at small values of $v_{+}^{(\mathrm{n})}$. Thus the UCNs most in danger of suffering depolarization are confined to a narrow space of $\sim 10 \mathrm{~cm}$ about the neutral plane, moving laterally as they float in the field (for $v_{+}^{(\mathrm{n})}=0$ ) or oscillate up and down about the neutral plane with small amplitude.

The magnetic field configurations and neutron orbits in actual or projected 3D magneto-gravitational UCN confinement systems [11-15] are more complex than in our field model. However, for some of these concepts the 1D approximation of our model appears to be justified. For the "bathtub" system [14], the neutral plane of our model, about which low-energy UCNs oscillate, corresponds to a strongly anisotropic oscillator potential in the vicinity of the minimum of potential $g y+\left|\mu_{n}\right| \frac{B}{m}$ for the low-field seeking spin state. For $B_{10}=0.005 \mathrm{~T}$ the minimum is located about $1.8 \mathrm{~cm}$ up from the lowest point of the double-curved surface of permanent magnets of Ref. [14]. For this anisotropic oscillator, the frequency for oscillations in the vertical $y$-direction, $\omega_{0 y}=$ $\left(\frac{d g_{+}}{d y}\right)^{1 / 2}=57 \mathrm{~s}^{-1}$, is 22 times larger than for the $z$ direction, $\omega_{0 z}=\left(\frac{g}{R_{z}}\right)^{1 / 2}=2.6 \mathrm{~s}^{-1}$ where the radius of curvature is $R_{z}=\rho=1.5 \mathrm{~m}$. For the $x$ direction, with its asymmetry, there are two curvatures $(0.5$ and $1.0 \mathrm{~m})$ and, therefore, two ratios replacing 22: 13 on one side $(x<0)$ and 18 for $x>0$. Since all these factors are large, the neutrons move almost freely, on a relative scale, in the peripheral $x$ and $z$ directions and, therefore, our 1D model should be a good approximation. As a result, we expect the peripheral velocity in the plane of the Halbach field, which corresponds to $v_{x}$ in the model, to be the main source of depolarization.

In the cylindrical field geometries of references [11-13,15], the field magnitude $B$ varies more slowly in space. Thus, the oscillator is less anisotropic, the degrees of freedom of motion in different coordinate directions are less decoupled and a more complex analysis may be required. To the extent that qualitative features of our model may still apply we expect that the main source of depolarization would be a large peripheral UCN velocity perpendicular to the cylinder axis. In this case, the peripheral velocity corresponds to the component $v_{x}$ of the model.

Besides depolarization of magnetically confined UCNs, we also studied depolarization in UCN reflection on a nonmagnetic mirror immersed in a magnetic field. The field was our model field into which we conceptually inserted an ideal neutron mirror horizontally at a variable height. The problem of possible depolarization in mirror reflection is of paramount importance in UCN experiments on the asymmetry parameter $A$ in neutron decay $[20,21]$ and it is also encountered in high-field UCN polarizers. Depolarization is expected since the adiabaticity condition may be violated due to the abrupt change of flight direction at the reflection point, thus $d \mathbf{B} / d t$ changes abruptly. This problem has first been studied in Ref. [17] by adapting the Majorana semi-classical approach [18] to the mirror geometry. In our analysis the three methods used (quantum approximation, semi-classical and numerical approach) gave identical results for depolarization in magnetic UCN storage. For the mirror reflection problem the quantum result (52) and the semiclassical result (68) are very similar but not identical, as shown in Fig. 5. The numerical method is not accurate enough in this case to distinguish between the two. The semiclassical result (68) lacks the factor $k_{-m} / k_{+m}$ which would cause a divergence if the mirror is placed at a turning point height (since $k_{+}=0$ in this region).

Between the turning points the difference is minor and the common result is as follows: Depolarization in mirror reflection, averaged over field directions as in our model field, is determined mainly by the second term of Eq. (68). It increases with the frequency of field variation $\left(\sim K k_{x}\right)$ seen by the neutron moving along the in-plane $x$ direction. It also increases quadratically with the sine of the field angle $\theta$, which is a measure of Halbach field strength $B_{H}=B \sin \theta$, and with its gradient, $\theta^{\prime}=\frac{d \theta}{d y}$, at the mirror location. Depolarization strongly decreases with increasing field strength $B$ at the mirror $\left(\sim B_{H}^{2} / B^{4}\right)$. Figure 5 shows that, for $v_{x}=3 \mathrm{~m} / \mathrm{s}$, the magnitude of depolarization per one bounce on the mirror is up to $\sim 10^{4}$ times larger than the depolarization per bounce in the magnetic field without the mirror. The depolarization on the mirror has its peak value at the vertical location where the depolarization probability plotted in Fig. 2 also has its peak. In fact, comparing expression (68) for depolarization at the mirror with the result (34) for the field alone [and neglecting the small first term on the right-hand side of Eq. (68)] we realize that the mirror acts like a polarization analyzer inserted into the particle beam moving through the $\mathbf{B}$ field. This interpretation also holds for a nonhorizontal or curved mirror since the second term on the right-hand side of Eq. (68) is independent of the orientation of the reflecting surface element. In this case, the second term should be averaged over the mirror extension.

In cases where many successive wall reflections take place in a weak, nonuniform magnetic field, the depolarization may become significant. Comparing our result (66) with equations (10 and 11) of Ref. [17] we note that both results have the square of the Larmor frequency $\omega_{L}$ at the mirror position in the denominator. However, a quantitative comparison is difficult since the magnetic field variations assumed in the two approaches are different. In either analysis, no depolarization is expected for a uniform magnetic field.

Our field model may be too specific to allow a quantitative comparison with the data $[25,26]$ on depolarization in UCN reflection from various mirror materials (like beryllium or samples with diamond-like carbon coating). Expression (68) does not depend on specific properties of the mirror, as long as it is a good, nearly loss-free UCN reflector. Therefore, Eq. (68) could explain, without having to invoke any anomalies [25], the remarkable similarity and temperature independence of depolarization probabilities measured for different wall materials. Such independence would be expected if the samples were exposed to the same nonuniform magnetic field.

Our results, which were obtained as straightforward solutions to the spin-dependent Schrödinger equation, may also 
provide an alternative to the discussion of new short-range, spin-dependent forces as a possible pathway to explaining the depolarization data for stored UCNs. For a recent comprehensive review of fundamental physics with neutrons see Ref. [27].

\section{ACKNOWLEDGMENTS}

We thank V. Ezhov, C. Liu, and A. Young for having directed our attention to the question of depolarization in magnetic UCN confinement.
[1] K. Nakamura et al. (Particle Data Group), J. Phys. G 37, 075021 (2010), and 2012 partial update.

[2] A. Serebrov et al., Phys. Lett. B 605, 72 (2005); Phys. Rev. C 78, 035505 (2008).

[3] W. Mampe, P. Ageron, C. Bates, J. M. Pendlebury, and A. Steyerl, Phys. Rev. Lett. 63, 593 (1989); and update, Ref. [9].

[4] W. Mampe, L. N. Bondarenko, V. I. Morozov, Yu. N. Panin, and A. I. Fomin, JETP Lett. 57, 82 (1993).

[5] J. Byrne et al., Eur. Phys. Lett. 33, 187 (1996).

[6] S. S. Arzumanov et al., Nucl. Instrum. Methods Phys. Res. A 440, 511 (2000); JETP Lett. 95, 224 (2012).

[7] J. S. Nico et al., Phys. Rev. C 71, 055502 (2005).

[8] A. Pichlmaier, V. Varlamov, K. Schreckenbach, and P. Geltenbort, Phys. Lett. B 693, 221 (2010).

[9] A. Steyerl, J. M. Pendlebury, C. Kaufman, S. S. Malik, and A. M. Desai, Phys. Rev. C 85, 065503 (2012).

[10] W. Paul et al., Z. Phys. C 45, 25 (1989).

[11] V. F. Ezhov et al., J. Res. Natl. Inst. Stand. Technol. 110, 345 (2005); Nucl. Instrum. Methods Phys. Res. A 611, 167 (2009).

[12] K. Leung and O. Zimmer, Nucl. Instrum. Methods Phys. Res. A 611, 181 (2009).
[13] P. R. Huffman et al., Nature 403, 62 (2000).

[14] P. L. Walstrom et al., Nucl. Instrum. Methods Phys. Res. A 599, 82 (2009).

[15] S. Materne et al., Nucl. Instrum. Methods Phys. Res. A 611, 176 (2009).

[16] V. V. Vladimirsky, JETP 12, 740 (1961).

[17] Yu. N. Pokotilovski, JETP Lett. 76, 131 (2002); 78, 422(E) (2003).

[18] E. Majorana, Il Nuovo Cimento 9, 43 (1932).

[19] J. C. Mallinson, IEEE Trans. Magn. 9, 1 (1973).

[20] R. W. Pattie et al., Phys. Rev. Lett. 102, 012301 (2009).

[21] J. Liu et al., Phys. Rev. Lett. 105, 181803 (2010).

[22] A. Messiah, Quantum Mechanics (Wiley, New York, 1961).

[23] P. M. Morse and H. Feshbach, Methods of Theoretical Physics (McGraw-Hill, New York, 1953), Chap. 9.3.

[24] M. Abramowitz and I. A. Stegun, Handbook of Mathematical Functions (Dover, New York, 1970), Chap. 10.4.

[25] A. P. Serebrov et al., Phys. Lett. A 313, 373 (2003); 335, 327 (2005).

[26] F. Atchison et al., Phys. Rev. C 76, 044001 (2007).

[27] D. Dubbers and M. G. Schmidt, Rev. Mod. Phys. 83, 1111 (2011). 